\title{
A SINGULAR CONTROLLABILITY PROBLEM WITH VANISHING VISCOSITY
}

\author{
IOAN Florin Bugariu ${ }^{1}$ AND SORIN MiCU ${ }^{1}$
}

\begin{abstract}
The aim of this paper is to answer the question: Do the controls of a vanishing viscosity approximation of the one dimensional linear wave equation converge to a control of the conservative limit equation? The characteristic of our viscous term is that it contains the fractional power $\alpha$ of the Dirichlet Laplace operator. Through the parameter $\alpha$ we may increase or decrease the strength of the high frequencies damping which allows us to cover a large class of dissipative mechanisms. The viscous term, being multiplied by a small parameter $\varepsilon$ devoted to tend to zero, vanishes in the limit. Our analysis, based on moment problems and biorthogonal sequences, enables us to evaluate the magnitude of the controls needed for each eigenmode and to show their uniform boundedness with respect to $\varepsilon$, under the assumption that $\alpha \in[0,1) \backslash\left\{\frac{1}{2}\right\}$. It follows that, under this assumption, our starting question has a positive answer.
\end{abstract}

Mathematics Subject Classification. 93B05, 30E05, 35E20.

Received June 6, 2012. Revised February 27, 2013.

Published online December 10, 2013.

\section{INTRODUCTION}

For $T>0$, we consider the one-dimensional linear wave equation with "lumped" control

$$
\begin{cases}w_{t t}(t, x)-\partial_{x x}^{2} w(t, x)=v(t) f(x) & (t, x) \in(0, T) \times(0, \pi) \\ w(t, 0)=w(t, \pi)=0 & t \in(0, T) \\ w(0, x)=w^{0}(x), w_{t}(0, x)=w^{1}(x) & x \in(0, \pi),\end{cases}
$$

where the profile $f \in L^{2}(0, \pi)$ is given and verifies $\widehat{f}_{n} \neq 0$ for every $n \geq 1$. Here and in the sequel, given any function $g \in L^{2}(0, \pi)$, we denote by $\widehat{g}_{n}$ the $n$-th Fourier coefficient of $g$,

$$
\widehat{g}_{n}=\int_{0}^{\pi} g(x) \sin (n x) \mathrm{d} x \quad(n \geq 1) .
$$

Equation (1.1) is said to be null-controllable in time $T>0$ if, for every initial data $\left(w^{0}, w^{1}\right) \in \mathcal{H}_{0} \subset H_{0}^{1}(0, \pi) \times$ $L^{2}(0, \pi)$, there exists a control $v \in L^{2}(0, T)$ such that the corresponding solution of (1.1) verifies

$$
w(T, \cdot)=w_{t}(T, \cdot)=0
$$

Keywords and phrases. Wave equation, null-controllability, vanishing viscosity, moment problem, biorthogonals.

1 Facultatea de Stiinte Exacte, Universitatea din Craiova, 200585, Romania.

florin_bugariu_86@yahoo.com; sd_micu@yahoo.com 
where the space $\mathcal{H}_{0}$ is defined as follows

$$
\mathcal{H}_{0}=\left\{\left(w^{0}, w^{1}\right) \in H_{0}^{1}(0, \pi) \times L^{2}(0, \pi) \mid \sum_{n \geq 1} \frac{n^{2}\left|\widehat{w}_{n}^{0}\right|^{2}+\left|\widehat{w}_{n}^{1}\right|^{2}}{\left|\widehat{f}_{n}\right|^{2}}<\infty\right\} .
$$

The goal is to drive the initial data $\left(w^{0}, w^{1}\right)$ to rest by using a control $v(t)$, depending only on time and acting on the system through a given shape function in space $f(x)$. Such types of controls are often used and sometimes called "lumped" or "bilinear" (see, for instance, $[1,12,21]$ ).

The controllability properties of (1.1) are by now well-known (see, for instance, the monographs $[7,34]$ ). One of the oldest methods used to study such controllability problems consists in reducing them to a moment problem whose solution is given in terms of an explicit biorthogonal sequence to a family $\Lambda$ of exponential functions. For instance, this method was used by Fattorini and Russell in the pioneering articles $[12,13]$ to prove the controllability of the one dimensional heat equation. In their case, the family $\Lambda$ has only real exponential functions. On the contrary, when equation (1.1) comes into discussion, the family $\Lambda$ is given by $\left(\mathrm{e}^{\mu_{n} t}\right)_{n \in \mathbb{Z}^{*}}$, where $\mu_{n}=i n, n \in \mathbb{Z}^{*}$, are the eigenvalues of the wave operator $\left(\begin{array}{cc}0 & -I \\ -\partial_{x x}^{2} & 0\end{array}\right)$ and are purely imaginary. It follows easily that, (1.1) is null-controllable in time $T$ if, and only if, for every initial data $\left(w^{0}, w^{1}\right) \in \mathcal{H}_{0}$, there exists a solution $v \in L^{2}(0, T)$ of the following moment problem:

$$
\int_{-\frac{T}{2}}^{\frac{T}{2}} v\left(t+\frac{T}{2}\right) \mathrm{e}^{\bar{\mu}_{n} t} \mathrm{~d} t=-\frac{\mathrm{e}^{-\frac{T}{2} \bar{\mu}_{n}}}{\widehat{f}_{|n|}}\left(\widehat{w}_{|n|}^{1}+\mu_{n} \widehat{w}_{|n|}^{0}\right) \quad\left(n \in \mathbb{Z}^{*}\right) .
$$

In order to fix some ideas and to illustrate the method used in this paper, let us briefly show how do we obtain a solution of (1.4). We begin by defining the function

$$
\widetilde{\Psi}_{m}(z)=\frac{\sin (\pi(z+m))}{\pi(z+m)}
$$

which is an entire function of exponential type $\pi$ such that $\int_{\mathbb{R}}\left|\widetilde{\Psi}_{m}(x)\right|^{2} \mathrm{~d} x<\infty$. It results from Paley-Wiener Theorem that the Fourier transform of $\widetilde{\Psi}_{m}$,

$$
\widetilde{\theta}_{m}(t)=\frac{1}{2 \pi} \int_{\mathbb{R}} \widetilde{\Psi}_{m}(x) \mathrm{e}^{-i x t} \mathrm{~d} x \quad\left(m \in \mathbb{Z}^{*}\right)
$$

belongs to $L^{2}(-\pi, \pi)$. Moreover, from the inversion formula, it follows that $\left(\widetilde{\theta}_{m}\right)_{m \in \mathbb{Z}^{*}}$ forms a biorthogonal sequence to the family $\Lambda=\left(\mathrm{e}^{\mu_{n} t}\right)_{n \in \mathbb{Z}^{*}}$, i.e. verify

$$
\int_{-\pi}^{\pi} \widetilde{\theta}_{m}(t) \mathrm{e}^{\bar{\mu}_{n} t} \mathrm{~d} t=\delta_{\mathrm{mn}} \quad\left(m, n \in \mathbb{Z}^{*}\right) .
$$

From the above properties, we deduce that a formal solution of the moment problem (1.4) is given by

$$
v(t)=-\sum_{m \in \mathbb{Z}^{*}} \frac{\mathrm{e}^{-\pi \bar{\mu}_{m}}}{\widehat{f}_{|m|}}\left(\widehat{w}_{|m|}^{1}+\mu_{m} \widehat{w}_{|m|}^{0}\right) \tilde{\theta}_{m}(t-\pi) \quad(t \in(0,2 \pi)) .
$$

In fact (1.8) gives a true solution of (1.4) if the right hand side of (1.8) converges in $L^{2}(0,2 \pi)$. For each $\left(w^{0}, w^{1}\right) \in \mathcal{H}_{0}$, the convergence of this series follows from the existence of a constant $C>0$ such that

$$
\left\|\widetilde{\theta}_{m}\right\|_{L^{2}(-\pi, \pi)} \leq C \quad\left(m \in \mathbb{Z}^{*}\right),
$$


which is a consequence of the uniform boundedness (in $m$ ) of the $L^{2}(\mathbb{R})-$ norms of $\left(\widetilde{\Psi}_{m}\right)_{m \in \mathbb{Z}^{*}}$ and Plancherel's Theorem. Hence, for any initial data $\left(w^{0}, w^{1}\right) \in \mathcal{H}_{0}$, the moment problem has at least a solution $v \in L^{2}(0,2 \pi)$, given by (1.8), and the controllability of (1.1) in time $T=2 \pi$ follows.

In many applications it is of interest to study the uniform controllability properties of (1.1) when a viscous term is introduced in the equation. Indeed, the mechanism of vanishing viscosity is a common tool in the study of Cauchy problems or in improving convergence of numerical schemes for hyperbolic conservation laws and shocks. For instance, in $[16,17]$, it is proved that, by adding an extra numerical viscosity term, the dispersive properties of the finite difference scheme for the nonlinear Schrödinger equation become uniform when the meshsize tends to zero. This scheme reproduces at the discrete level the properties of the continuous Schrödinger equation by dissipating the high frequency numerical spurious solutions. On the other hand, a viscosity term is introduced in [10] to prove the existence of solutions of hyperbolic equations. In both examples the viscosity is devoted to tend to zero in order to recover the original system. Thus, a legitimate question is related to the behavior and the sensitivity of the controls during this process. For instance, given $T>0$ and $\varepsilon \in(0,1)$, one could consider the perturbed wave equation

$$
\begin{cases}u_{t t}(t, x)-\partial_{x x}^{2} u(t, x)+2 \varepsilon\left(-\partial_{x x}^{2}\right)^{\alpha} u_{t}(t, x)=v_{\varepsilon}(t) f(x) & (t, x) \in(0, T) \times(0, \pi) \\ u(t, 0)=u(t, \pi)=0 & t \in(0, T) \\ u(0, x)=u^{0}(x), u_{t}(0, x)=u^{1}(x) & x \in(0, \pi)\end{cases}
$$

and study the possibility of obtaining a control $v$ of (1.1) as limit of controls $v_{\varepsilon} \in L^{2}(0, T)$ of (1.10). Here and in what follows $\left(-\partial_{x x}^{2}\right)^{\alpha}$ denotes the fractional power of order $\alpha \geq 0$ of the Dirichlet Laplace operator in $(0, \pi)$. More precisely,

$$
\begin{gathered}
\left(-\partial_{x x}^{2}\right)^{\alpha}: D\left(\left(-\partial_{x x}^{2}\right)^{\alpha}\right) \subset L^{2}(0, \pi) \rightarrow L^{2}(0, \pi), \\
D\left(\left(-\partial_{x x}^{2}\right)^{\alpha}\right)=\left\{u \in L^{2}(0, \pi): u=\sum_{n \geq 1} a_{n} \sin (n x) \text { and } \sum_{n \geq 1}\left|a_{n}\right|^{2} n^{4 \alpha}<\infty\right\}, \\
u(x)=\sum_{n \geq 1} a_{n} \sin (n x) \longrightarrow\left(-\partial_{x x}^{2}\right)^{\alpha} u(x)=\sum_{n \geq 1} a_{n} n^{2 \alpha} \sin (n x) .
\end{gathered}
$$

Equation (1.10) is dissipative and it can be easily checked that, if $f=0$,

$$
\frac{\mathrm{d}}{\mathrm{d} t}\left(\|u(t)\|_{H_{0}^{1}}^{2}+\left\|u_{t}(t)\right\|_{L^{2}}^{2}\right)=-2 \varepsilon \int_{0}^{\pi}\left|\left(-\partial_{x x}^{2}\right)^{\frac{\alpha}{2}} u_{t}(t, x)\right|^{2} \mathrm{~d} t \leq 0 .
$$

Hence, $2 \varepsilon\left(-\partial_{x x}^{2}\right)^{\alpha} u_{t}(t, x)$ represents an added viscous term devoted to vanish as $\varepsilon$ tends to zero. However, the controllability properties of (1.10) are poor. Indeed, the family of exponential functions corresponding to this case is given by $\Lambda=\left(\mathrm{e}^{\nu_{n} t}\right)_{n \in \mathbb{Z}^{*}}$, where $\nu_{n}=\varepsilon|n|^{2 \alpha}+\operatorname{sgn}(\mathrm{n}) \sqrt{|n|^{4 \alpha}-n^{2}}$. If $\alpha>\frac{1}{2}$, we have that

$$
\lim _{n \rightarrow-\infty} \nu_{n}=0,
$$

which implies that the family $\Lambda$ is not minimal. Consequently, equation (1.10) is not spectrally controllable if $\alpha>\frac{1}{2}$ (for more details in the case $\alpha=1$, see [30]).

Since we want to allow stronger dissipative terms which correspond to the case $\alpha>\frac{1}{2}$, we perturb the wave equation (1.1) in the following slightly different way

$$
\begin{cases}u_{t t}(t, x)-\partial_{x x}^{2} u(t, x)+2 \varepsilon\left(-\partial_{x x}^{2}\right)^{\alpha} u_{t}(t, x)+\varepsilon^{2}\left(-\partial_{x x}^{2}\right)^{2 \alpha} u(t, x)=v_{\varepsilon}(t) f(x) & (t, x) \in(0, T) \times(0, \pi) \\ u(t, 0)=u(t, \pi)=0 & t \in(0, T) \\ u(0, x)=u^{0}(x), u_{t}(0, x)=u^{1}(x) & x \in(0, \pi) .\end{cases}
$$


Equation (1.13) is still dissipative. Indeed, if $f=0$, we have that

$$
\frac{\mathrm{d}}{\mathrm{d} t}\left(\|u(t)\|_{H_{0}^{1}}^{2}+\varepsilon^{2}\left\|\left(-\partial_{x x}\right)^{\alpha} u(t)\right\|_{L^{2}}^{2}+\left\|u_{t}(t)\right\|_{L^{2}}^{2}\right)=-2 \varepsilon \int_{0}^{\pi}\left|\left(-\partial_{x x}^{2}\right)^{\frac{\alpha}{2}} u_{t}(t, x)\right|^{2} \mathrm{~d} t \leq 0 .
$$

Note that, if $\alpha \leq \frac{1}{2}$, the norm $\sqrt{\|u\|_{H_{0}^{1}}^{2}+\varepsilon^{2}\left\|\left(-\partial_{x x}\right)^{\alpha} u\right\|_{L^{2}}^{2}+\left\|u_{t}\right\|_{L^{2}}^{2}}$ is equivalent to $\sqrt{\|u\|_{H_{0}^{1}}^{2}+\left\|u_{t}\right\|_{L^{2}}^{2}}$ and the controllability properties of (1.10) and (1.13) are similar. However, in the case $\alpha>\frac{1}{2}$, the controllability properties of (1.13) are better than those of (1.10). Hence, the term $\varepsilon^{2}\left(-\partial_{x x}\right)^{2 \alpha} u(t, x)$ allows us to consider stronger dissipation and also to simplify some of our estimates.

The aim of this paper is to study the controllability properties of (1.13) and their relation with the ones of (1.1). The controllability of (1.13) is defined in a similar way as for (1.1). More precisely, given $T>0$ and $f \in L^{2}(0, \pi)$ with $\widehat{f}_{n} \neq 0$ for $n \geq 1$, equation (1.13) is null-controllable in time $T$ if, for any $\left(u^{0}, u^{1}\right) \in \mathcal{H}_{0}$, there exists a control $v_{\varepsilon} \in L^{2}(0, T)$ such that the corresponding solution $\left(u, u_{t}\right)$ of $(1.13)$ verifies

$$
u(T, \cdot)=u_{t}(T, \cdot)=0 .
$$

The null-controllability problem is equivalent to find, for every initial data $\left(u^{0}, u^{1}\right) \in \mathcal{H}_{0}$, a solution $v_{\varepsilon} \in L^{2}(0, T)$ of the following moment problem:

$$
\int_{-\frac{T}{2}}^{\frac{T}{2}} v_{\varepsilon}\left(t+\frac{T}{2}\right) \mathrm{e}^{\bar{\lambda}_{n} t} \mathrm{~d} t=-\frac{\mathrm{e}^{-\bar{\lambda}_{n} \frac{T}{2}}}{\widehat{f}_{|n|}}\left(\widehat{u}_{|n|}^{1}+\lambda_{n} \widehat{u}_{|n|}^{0}\right) \quad\left(n \in \mathbb{Z}^{*}\right),
$$

where $\lambda_{n}=i n+\varepsilon|n|^{2 \alpha}$, are the eigenvalues of the operator $\left(\begin{array}{cc}0 & -I \\ -\partial_{x x}^{2}+\varepsilon^{2}\left(-\partial_{x x}^{2}\right)^{2 \alpha} 2 \varepsilon\left(-\partial_{x x}^{2}\right)^{\alpha}\end{array}\right)$ corresponding to the "adjoint" problem of (1.13).

As in (1.4), if we have at our disposal a biorthogonal sequence to the family $\left(\mathrm{e}^{\lambda_{n} t}\right)_{n \in \mathbb{Z}^{*}}$, denoted by $\left(\theta_{m}\right)_{m \in \mathbb{Z}^{*}}$, we can give immediately a formal solution of (1.16),

$$
v_{\varepsilon}(t)=-\sum_{m \in \mathbb{Z}^{*}} \frac{\mathrm{e}^{-\bar{\lambda}_{m} \frac{T}{2}}}{\widehat{f}_{|m|}}\left(\widehat{u}_{|m|}^{1}+\lambda_{m} \widehat{u}_{|m|}^{0}\right) \theta_{m}\left(t-\frac{T}{2}\right) \quad(t \in(0, T)) .
$$

This time the family $\left(\mathrm{e}^{\lambda_{n} t}\right)_{n \in \mathbb{Z}^{*}}$ has no longer purely imaginarily exponents like in (1.1). Thus, it is not so easy as for (1.1) to give explicit entire functions $\left(\Psi_{m}\right)_{m \in \mathbb{Z}^{*}}$ whose Fourier transforms define a biorthogonal sequence $\left(\theta_{m}\right)_{m \in \mathbb{Z}^{*}}$ to $\left(\mathrm{e}^{\lambda_{n} t}\right)_{n \in \mathbb{Z}^{*}}$. Moreover, we cannot guarantee anymore the boundedness of the sequence $\left(\theta_{m}\right)_{m \in \mathbb{Z}^{*}}$ and (1.9) will be replaced by an estimate of the form

$$
\left\|\theta_{m}\right\|_{L^{2}} \leq C \mathrm{e}^{\beta\left|\Re\left(\lambda_{m}\right)\right|} \quad\left(m \in \mathbb{Z}^{*}\right) .
$$

Note that $\left\|\theta_{m}\right\|_{L^{2}}$ may become exponentially large as $m$ goes to infinity. By taking into account the damping mechanism introduced in equation (1.13), this growth estimate guarantees the convergence of series (1.17) for each initial data $\left(u^{0}, u^{1}\right) \in \mathcal{H}_{0}$, if $T$ is large enough. However, in order show that a control time $T$ independent of $\varepsilon$ can be chosen and to prove the boundedness of the family of controls $\left(v_{\varepsilon}\right)_{\varepsilon \in(0,1)}$ in $L^{2}(0, T)$, the dependence in $\varepsilon$ of the constants $C$ and $\beta$ from (1.18) is required. This represents one of the most difficult tasks of our work. We shall prove that $C$ and $\beta$ from (1.18) can be chosen independent of $\varepsilon$, fact that ensures the uniform boundedness of the sequence $\left(v_{\varepsilon}\right)_{\varepsilon \in(0,1)}$ and the possibility to pass to the limit as $\varepsilon$ tends to zero in (1.13). The main result of this paper reads as follows.

Theorem 1.1. Let $\alpha \in[0,1) \backslash\left\{\frac{1}{2}\right\}$ and $f \in L^{2}(0, \pi)$ be a function such that $\widehat{f}_{n} \neq 0$ for every $n \geq 1$. There exists a time $T>0$ with the property that, for any $\left(u^{0}, u^{1}\right) \in \mathcal{H}_{0}$ and $\varepsilon \in(0,1)$, there exists a control $v_{\varepsilon} \in L^{2}(0, T)$ of (1.13) such that the family $\left(v_{\varepsilon}\right)_{\varepsilon \in(0,1)}$ is uniformly bounded in $L^{2}(0, T)$ and any weak limit $v$ of it, as $\varepsilon$ tends to zero, is a control in time $T$ for equation (1.1). 
The controllability problem studied in this paper belongs to the interface between parabolic and hyperbolic equations. From this point of view, it is related to $[8,14,23]$, where the controllability of the transport equation is addressed after the introduction of a vanishing viscosity term. In [8] Carleman estimates are used to obtain an uniform bound for the family of controls. The same result is shown in [14], improving the control time, by means of nonharmonic Fourier analysis and biorthogonal technique. The recent article [23] deals with a nonlinear scalar conservation law perturbed by a small viscosity term and proves the uniform boundedness of the boundary controls. Related problems in which controls for an equation are obtained as limits of controls of equations of different type may be also found in [24, 26, 28,31,37].

In order to justify the damping mechanism introduced in (1.13), which involves the fractional power $\alpha$ of the Laplace operator, let us point out that sometimes it may be useful to control the amount of dissipation introduced in the system not only by means of the vanishing parameter $\varepsilon$ but also by an adequate choice of the differential operator. For instance, the convergence rates in some perturbed problems can be improved by choosing a viscosity operator of lower order (see, for instance, [18] in the context of Hamilton-Jacobi equations). In (1.13) this is achieved through the parameter $\alpha$. The case $\alpha=1$ has been studied, for a slightly different problem, in [25], where a uniform controllability result with respect to the viscosity is proved. Theorem 1.1 shows that a similar result holds for any $\alpha \in[0,1) \backslash\left\{\frac{1}{2}\right\}$. Note that, if $\alpha \in\left[0, \frac{1}{2}\right)$, the imaginary parts of the eigenvalues $\lambda_{n}$ dominate the real ones and problem (1.13) has the same hyperbolic character as in the limit case $\varepsilon=0$. On the contrary, if $\alpha \in\left(\frac{1}{2}, 1\right),(1.13)$ has a parabolic type. In this case we are dealing with a truly singular control problem and the pass to the limit is sensibly more difficult. Finally, let us remark that $\alpha=\frac{1}{2}$ is a singular case in which the basic controllability properties (such as spectral controllability) of (1.13) do not hold.

For $\alpha \in[0,1) \backslash\left\{\frac{1}{2}\right\}$, the construction from the proof of Theorem 1.1 implies that the following Ingham-type inequality (see [20]) holds, for any finite sequence $\left(\beta_{n}\right)_{n \in \mathbb{Z}^{*}}$ and $T$ sufficiently large,

$$
C(T, \alpha) \sum_{n \in \mathbb{Z}^{*}}\left|\beta_{n}\right|^{2} \mathrm{e}^{-\omega \varepsilon|n|^{2 \alpha}} \leq \int_{-T}^{T}\left|\sum_{n \in \mathbb{Z}^{*}} \beta_{n} \mathrm{e}^{\lambda_{n} t}\right|^{2} \mathrm{~d} t,
$$

where $\varepsilon \in(0,1), \omega$ is an absolute positive constant and $C$ a positive constant depending of $T$ and $\alpha$ but independent of $\varepsilon$. From this point of view our article extends the results from $[11,15,32]$, where Ingham-type inequalities are obtained under a more restrictive uniform sparsity condition of the sequence $\left(\lambda_{n}\right)_{n \in \mathbb{Z}^{*}}$. Indeed, one of the major difficulty in our study is related to the fact that the sequence of our eigenvalues $\left(\lambda_{n}\right)_{n \in \mathbb{Z}^{*}}$ is not included in a sector of the positive real axis and does not verify a uniform separation condition of the type

$$
\left|\lambda_{n}-\lambda_{m}\right| \geq \delta\left|n^{\beta}-m^{\beta}\right| \quad\left(n, m \in \mathbb{Z}^{*}\right),
$$

for some $\beta>1$ and $\delta>0$ independent of $\varepsilon$. The fact that $C(T, \alpha)$ in (1.19) does not depend of $\varepsilon$ is of fundamental importance since it ensures the uniform boundedness of a family of controls $\left(v_{\varepsilon}\right)_{\varepsilon \in(0,1)}$ for $(1.13)$ and the possibility to pass to the limit in order to obtain a control $v$ for (1.1).

The rest of the paper is organized as follows. Section 2 gives the equivalent characterization of the controllability property in terms of a moment problem. The core of the paper is Section 3 where two biorthogonal sequences to the family $\left(\mathrm{e}^{\lambda_{n} t}\right)_{n \in \mathbb{Z}^{*}}$ are constructed and evaluated. The proof of Theorem 1.1 is provided in Section 4. The article ends with an Appendix in which a technical lemma is proved.

\section{THE MOMENT PROBLEM}

In this section we show the equivalence between the controllability problem (1.13)-(1.15) and the moment problem (1.16). In order to do this we need first a result concerning the existence of solutions for equation (1.13). More precisely we have the following property. 
Proposition 2.1. Given any $T>0, \alpha \in[0,1], \varepsilon \geq 0, h \in L^{1}\left(0, T ; L^{2}(0, \pi)\right)$ and $\left(u^{0}, u^{1}\right) \in H_{0}^{1}(0, \pi) \times L^{2}(0, \pi)$, there exists a unique weak solution $\left(u, u_{t}\right) \in C\left([0, T], H_{0}^{1}(0, \pi) \times L^{2}(0, \pi)\right)$ of the problem

$$
\begin{cases}u_{t t}+\left(-\partial_{x x}^{2}\right) u+\varepsilon^{2}\left(-\partial_{x x}^{2}\right)^{2 \alpha} u+2 \varepsilon\left(-\partial_{x x}^{2}\right)^{\alpha} u_{t}=h(t, x) & (x, t) \in(0, \pi) \times(0, T) \\ u(t, 0)=u(t, \pi)=0 & t \in(0, T) \\ u(0, x)=u^{0}(x) & x \in(0, \pi) .\end{cases}
$$

Proof. For any $\alpha \in[0,1]$ and $\varepsilon \geq 0$, let

$$
\mathcal{X}= \begin{cases}D\left(\left(-\partial_{x x}^{2}\right)^{\alpha}\right) \times L^{2}(0, \pi) & \text { if } \varepsilon>0 \text { and } \alpha>\frac{1}{2} \\ H_{0}^{1}(0, \pi) \times L^{2}(0, \pi) & \text { if } \varepsilon=0 \text { or } \varepsilon>0 \text { and } \alpha \leq \frac{1}{2},\end{cases}
$$

(for the definition of the operator $\left(D\left(\left(-\partial_{x x}^{2}\right)^{\alpha}\right),\left(-\partial_{x x}^{2}\right)^{\alpha}\right)$, see (1.11)). We suppose that the space $\mathcal{X}$ is endowed with the inner product

$$
\left(\left(u_{1}, u_{2}\right),\left(v_{1}, v_{2}\right)\right)_{\mathcal{X}}=\left(\partial_{x} u_{1}, \partial_{x} v_{1}\right)_{L^{2}(0, \pi)}+\varepsilon^{2}\left(\left(-\partial_{x x}^{2}\right)^{\alpha} u_{1},\left(-\partial_{x x}^{2}\right)^{\alpha} v_{1}\right)_{L^{2}(0, \pi)}+\left(u_{2}, v_{2}\right)_{L^{2}(0, \pi)} .
$$

Note that, since $D\left(\left(-\partial_{x x}^{2}\right)^{1 / 2}\right)=H_{0}^{1}(0, \pi)$, it results that $\mathcal{X} \subseteq H_{0}^{1}(0, \pi) \times L^{2}(0, \pi)$. Now, we define the unbounded operator $(D(A), A)$ in $\mathcal{X}$ as follows,

$$
\begin{gathered}
D(A)=\left\{\begin{array}{l}
D\left(\left(-\partial_{x x}^{2}\right)^{2 \alpha}\right) \times D\left(\left(-\partial_{x x}^{2}\right)^{\alpha}\right) \quad \text { if } \varepsilon>0 \text { and } \alpha>\frac{1}{2} \\
H^{2}(0, \pi) \cap H_{0}^{1}(0, \pi) \times H_{0}^{1}(0, \pi) \text { if } \varepsilon=0 \text { or } \varepsilon>0 \text { and } \alpha \leq \frac{1}{2},
\end{array}\right. \\
A=\left(\begin{array}{cc}
0 & -I \\
-\partial_{x x}^{2}+\varepsilon^{2}\left(-\partial_{x x}^{2}\right)^{2 \alpha} 2 \varepsilon\left(-\partial_{x x}^{2}\right)^{\alpha}
\end{array}\right) .
\end{gathered}
$$

The operator $(D(A), A)$ is maximal and monotone in $\mathcal{X}$. Indeed, for any $U=\left(u_{1}, u_{2}\right) \in D(A)$, we have that

$$
\Re(A U, U)_{\mathcal{X}}=2 \varepsilon\left(\left(-\partial_{x x}^{2}\right)^{\frac{\alpha}{2}} u_{2},\left(-\partial_{x x}^{2}\right)^{\frac{\alpha}{2}} u_{2}\right)_{L^{2}(0, \pi)} \geq 0
$$

from which we deduce that $(D(A), A)$ is monotone in $\mathcal{X}$.

To prove that $(D(A), A)$ is maximal in $X$ we only have to show that there exists $\lambda>0$ with the property that for any $F=\left(f_{1}, f_{2}\right) \in \mathcal{X}$ there exits $U=\left(u_{1}, u_{2}\right) \in D(A)$ such that

$$
(A+\lambda I) U=F,
$$

which is equivalent to

$$
\left\{\begin{array}{l}
u_{2}=\lambda u_{1}-f_{1} \\
-\partial_{x x}^{2} u_{1}+\varepsilon^{2}\left(-\partial_{x x}^{2}\right)^{2 \alpha} u_{1}+\lambda^{2} u_{1}+2 \varepsilon \lambda\left(-\partial_{x x}^{2}\right)^{\alpha} u_{1}=f_{2}+2 \varepsilon\left(-\partial_{x x}^{2}\right)^{\alpha} f_{1}+\lambda f_{1} .
\end{array}\right.
$$

Nextly, we define the bilinear form $a: \widetilde{\mathcal{X}} \times \widetilde{\mathcal{X}} \rightarrow \mathbb{C}$ and the linear form $L: \widetilde{\mathcal{X}} \rightarrow \mathbb{C}$ given by

$$
\begin{aligned}
a(\varphi, \psi)= & \int_{0}^{\pi} \varphi_{x}(x) \overline{\psi_{x}(x)} \mathrm{d} x+\varepsilon^{2} \int_{0}^{\pi}\left(-\partial_{x x}^{2}\right)^{\alpha} \varphi(x) \overline{\left(-\partial_{x x}^{2}\right)^{\alpha} \psi(x)} \mathrm{d} x \\
& +2 \varepsilon \lambda \int_{0}^{\pi}\left(-\partial_{x x}^{2}\right)^{\frac{\alpha}{2}} \varphi(x) \overline{\left(-\partial_{x x}^{2}\right)^{\frac{\alpha}{2}} \psi(x)} \mathrm{d} x+\lambda^{2} \int_{0}^{\pi} \varphi(x) \overline{\psi(x)} \mathrm{d} x \\
L(\psi)= & \int_{0}^{\pi}\left(f_{2}(x)+2 \varepsilon\left(-\partial_{x x}^{2}\right)^{\alpha} f_{1}(x)+\lambda f_{1}(x) \overline{\psi(x)} \mathrm{d} x\right.
\end{aligned}
$$


where

$$
\widetilde{\mathcal{X}}=\left\{\begin{array}{l}
D\left(\left(-\partial_{x x}^{2}\right)^{\alpha}\right) \text { if } \varepsilon>0 \text { and } \alpha>\frac{1}{2} \\
H_{0}^{1}(0, \pi) \quad \text { if } \varepsilon=0 \text { or } \varepsilon>0 \text { and } \alpha \leq \frac{1}{2} .
\end{array}\right.
$$

From Lax-Milgram Theorem it follows that the problem

$$
a(\varphi, \psi)=L(\psi) \quad(\psi \in \widetilde{\mathcal{X}})
$$

has a unique solution $\varphi \in \widetilde{\mathcal{X}}$. Consequently, (2.2) has a solution $U \in D(A)$ and the maximality of the operator $(D(A), A)$ follows. From Hille-Yosida's Theorem we deduce that the operator $(D(A), A)$ generates a semigroup of contraction $(S(t))_{t>0}$ in $\mathcal{X}$. At the same time, [4], Lemma 4.1.5 ensures the existence and uniqueness of a weak solution for (2.1) which belongs to $C([0, T], \mathcal{X})$. Since $\left.\mathcal{X} \subseteq H_{0}^{1}(0, \pi) \times L^{2}(0, \pi)\right)$, the proof is completed.

Remark 2.2. A more general equation than (2.1) is studied in $[5,6]$. It is proved that, if $\frac{1}{2} \leq \alpha \leq 1$, the generated semigroup is analytic on a triangular sector of $\mathbb{C}$ containing the positive real axis. This is not true if $0 \leq \alpha<\frac{1}{2}$.

Remark 2.3. A lumped control of the form $v_{\varepsilon}(t) f(x)$ has been chosen to act on our system (1.13). Of course, other types of controls can be proposed (interior, boundary etc.). At least formally, these new controllability problems may be reduced to prove an inequality of type (1.19) and the same technique could be used to study all of them. However, in the boundary control case we need to work in a space $\tilde{\mathcal{X}}$ not included in any $D\left(\left(-\partial_{x}^{2}\right)^{\alpha}\right)$, $\alpha>0$, which requires much more care. We recall that the fractional Laplace operator may also be introduced by considering the Dirichlet to Neumann map for the two-dimensional cylinder $(0, \pi) \times \mathbb{R}_{+}$(see $[2,3]$ for details). This method is commonly used in the recent literature since it allows to write nonlocal problems in a local way and this permits to use the variational techniques for these kind of problems. It is known that, for functions in the spaces $D\left(\left(-\partial_{x}^{2}\right)^{\alpha}\right)$ from above, this definition is coherent with the spectral one (1.11) used by us (see [2], Cor. 3.6). Consequently, homogeneous Dirichlet boundary condition may be easily treated by using the spectral definition of the fractional Laplace operator. On the contrary, to study the nonhomogeneous boundary problems for the same operator (or for general nonlocal operator) the strategy should be different. These problems are known to be more difficult and even ill-posed [9].

Now we can give the characterization of the controllability property of (1.13)-(1.15) in terms of a moment problem. Based on the Fourier expansion of solutions, the moment problems have been widely used in linear control theory. We refer to $[1,22,34,36]$ for a detailed discussion of the subject.

Theorem 2.4. Let $T>0, \varepsilon \in(0,1),\left(u^{0}, u^{1}\right) \in \mathcal{H}_{0}$ and $f \in L^{2}(0, \pi)$. There exists a control $v_{\varepsilon} \in L^{2}(0, T)$ such that the solution $\left(u, u_{t}\right)$ of equation (1.13) verifies (1.15), if and only if, $v_{\varepsilon} \in L^{2}(0, T)$ satisfies

$$
\widehat{f}_{|n|} \int_{-\frac{T}{2}}^{\frac{T}{2}} v_{\varepsilon}\left(t+\frac{T}{2}\right) \mathrm{e}^{\bar{\lambda}_{n} t} \mathrm{~d} t=-\mathrm{e}^{-\bar{\lambda}_{n} \frac{T}{2}}\left(\widehat{u}_{|n|}^{1}+\lambda_{n} \widehat{u}_{|n|}^{0}\right) \quad\left(n \in \mathbb{Z}^{*}\right),
$$

where $\lambda_{n}=i n+\varepsilon|n|^{2 \alpha}$, for any $n \in \mathbb{Z}^{*}$.

Proof. We consider the "adjoint" equation

$$
\left\{\begin{array}{lc}
\varphi_{t t}+\left(-\partial_{x x}^{2}\right) \varphi+\varepsilon^{2}\left(-\partial_{x x}^{2}\right)^{2 \alpha} \varphi-2 \varepsilon\left(-\partial_{x x}^{2}\right)^{\alpha} \varphi_{t}=0(x, t) \in(0, \pi) \times(0, T) \\
\varphi(t, 0)=\varphi(t, \pi)=0 & t \in(0, T) \\
\varphi(T, x)=\varphi^{0}(x) \quad \varphi_{t}(T, x)=\varphi^{1}(x) & x \in(0, \pi) .
\end{array}\right.
$$

If we multiply (1.13) by $\bar{\varphi}$ and we integrate by parts over $(0, T) \times(0, \pi)$, we deduce that $v_{\varepsilon} \in L^{2}(0, T)$ is a control for (1.13) if, and only if, it verifies

$$
\int_{0}^{T} v_{\varepsilon}(t) \int_{0}^{\pi} f(x) \bar{\varphi}(t, x) \mathrm{d} x \mathrm{~d} t=-\int_{0}^{\pi} u^{1}(x) \bar{\varphi}(0, x) \mathrm{d} x+\int_{0}^{\pi} u^{0}(x)\left(\bar{\varphi}_{t}(0, x)-2 \varepsilon\left(-\partial_{x x}^{2}\right)^{\alpha} \bar{\varphi}(0, x)\right) \mathrm{d} x,
$$


for every $\left(\varphi, \varphi_{t}\right)$ solution of $(2.5)$ with the initial data $\left(\varphi^{0}, \varphi^{1}\right)$. Since $(\sin (n x))_{n>1}$ is a basis for $L^{2}(0, \pi)$ we have to check (2.6) only for the initial data of the form $\left(\varphi^{0}, \varphi^{1}\right)=(\sin (n x), 0)$ and $\left(\varphi^{0}, \varphi^{1}\right)=(0, \sin (n x))$, for each $n \geq 1$. In the first case the solution of (2.6) is given by

$$
\varphi(t, x)=\left(\frac{\bar{\lambda}_{n}}{\bar{\lambda}_{n}-\lambda_{n}} \mathrm{e}^{(t-T) \lambda_{n}}+\frac{\lambda_{n}}{\lambda_{n}-\bar{\lambda}_{n}} \mathrm{e}^{(t-T) \bar{\lambda}_{n}}\right) \sin (n x) \quad\left(n \in \mathbb{N}^{*}\right),
$$

whereas in the second case it becomes

$$
\varphi(t, x)=\left(\frac{1}{\lambda_{n}-\bar{\lambda}_{n}} \mathrm{e}^{(t-T) \lambda_{n}}+\frac{1}{\bar{\lambda}_{n}-\lambda_{n}} \mathrm{e}^{(t-T) \bar{\lambda}_{n}}\right) \sin (n x) \quad\left(n \in \mathbb{N}^{*}\right) .
$$

By tacking in (2.6) $\varphi$ of the form (2.7) and (2.8), we obtained that $v_{\varepsilon} \in L^{2}(0, T)$ is a control of (1.13) if and only if it verifies (2.4).

Remark 2.5. Note that $\left(\lambda_{n}\right)_{n \in \mathbb{Z}^{*}}$ introduced in the previous theorem are the eigenvalues of the differential operator corresponding to the "adjoint" equation (2.5).

Remark 2.6. The condition $\widehat{f}_{n} \neq 0$ for any $n \in \mathbb{N}^{*}$ is necessary in order to solve the moment problem (2.4) for any initial data in $\mathcal{H}_{0}$. Indeed, if there exists $n_{0} \in \mathbb{N}^{*}$ such that $\widehat{f}_{n_{0}}=0$, then (2.4) has a solution only if the initial data $\left(u^{0}, u^{1}\right)$ verify the additional condition $\widehat{u}_{n_{0}}^{1}+\lambda_{n_{0}} \widehat{u}_{n_{0}}^{0}=0$.

We recall that $\left(\theta_{m}\right)_{m \in \mathbb{Z}^{*}} \in L^{2}\left(-\frac{T}{2}, \frac{T}{2}\right)$ is a biorthogonal sequence to the family of exponential functions $\left(\mathrm{e}^{\lambda_{n} t}\right)_{n \in \mathbb{Z}^{*}} \in L^{2}\left(-\frac{T}{2}, \frac{T}{2}\right)$ if and only if

$$
\int_{-\frac{T}{2}}^{\frac{T}{2}} \theta_{m}(t) \mathrm{e}^{\bar{\lambda}_{n} t} \mathrm{~d} t=\delta_{\mathrm{mn}} \quad\left(m, n \in \mathbb{Z}^{*}\right)
$$

It is easy to see from $(2.4)$ that, if $\left(\theta_{m}\right)_{m \in \mathbb{Z}^{*}}$ is a biorthogonal sequence to the family of exponential functions $\left(\mathrm{e}^{\lambda_{n} t}\right)_{n \in \mathbb{Z}^{*}}$ in $L^{2}\left(-\frac{T}{2}, \frac{T}{2}\right)$, then a control of $(1.13)$ is given by

$$
v_{\varepsilon}(t)=-\sum_{m \in \mathbb{Z}^{*}} \frac{\mathrm{e}^{-\bar{\lambda}_{m} \frac{T}{2}}}{\widehat{f}_{|m|}}\left(\widehat{u}_{|m|}^{1}+\lambda_{m} \widehat{u}_{|m|}^{0}\right) \theta_{m}\left(t-\frac{T}{2}\right) \quad(t \in(0, T)),
$$

provided that the right hand side converges in $L^{2}(0, T)$. Now the main problem is to show that there exists a biorthogonal sequence $\left(\theta_{m}\right)_{m \in \mathbb{Z}^{*}}$ to the family of exponential functions $\left(\mathrm{e}^{\lambda_{n} t}\right)_{n \in \mathbb{Z}^{*}}$ in $L^{2}\left(-\frac{T}{2}, \frac{T}{2}\right)$ and to evaluate its norm, in order to prove the convergence of the right hand side of (2.9) for any $\left(u^{0}, u^{1}\right) \in \mathcal{H}_{0}$.

\section{Construction of a Biorthogonal sequence}

The aim of this section is to construct and evaluate an explicit biorthogonal sequence to the family $\left(\mathrm{e}^{t \lambda_{n}}\right)_{n \in \mathbb{Z}^{*}}$ in $L^{2}\left(-\frac{T}{2}, \frac{T}{2}\right)$, where $\lambda_{n}=i n+\varepsilon|n|^{2 \alpha}$ are the eigenvalues introduced in Theorem 2.4. In order to do that, we define a family $\left(\Psi_{m}(z)\right)_{m \in \mathbb{Z}^{*}}$ of entire functions of exponential type independent of $\varepsilon$ (see, for instance, [35]) such that $\Psi_{m}\left(i \bar{\lambda}_{n}\right)=\delta_{\mathrm{mn}}$. The inverse Fourier transform of $\left(\Psi_{m}\right)_{m \in \mathbb{Z}^{*}}$ will give us the biorthogonal sequence $\left(\theta_{m}\right)_{m \in \mathbb{Z}^{*}}$ that we are looking for. Each $\Psi_{m}$ is obtained from a Weierstrass product $P_{m}$ multiplied by an appropriate function $M_{m}$ with rapid decay on the real axis. Such a method was used for the first time by Paley and Wiener [29] and, in the context of control problems, by Fattorini and Russell [12,13]. The main difficulty in our analysis is to obtain good estimates for the behavior of $P_{m}$ on the real axis and to construct an appropriate multiplier $M_{m}$ in order to ensure the boundedness of $\Psi_{m}$ on the real axis. As we shall see in Proposition 3.6 below, the behavior of $\ln \left|P_{m}(x)\right|$ is always dominated by a subunitary power of $|x|$, if $\alpha<\frac{1}{2}$ which facilitates 
the entire construction and analysis. In the more difficult case $\alpha>\frac{1}{2}, \ln \left|P_{m}(x)\right|$ behaves like $|x|$ on an interval of length $O\left(\left(\frac{1}{\varepsilon}\right)^{\frac{1}{2 \alpha-1}}\right)$. It is precisely this property which makes the construction of $M_{m}$ more problematic and imposes the necessity of a careful analysis of $P_{m}(x)$. Finally, the bounds obtained on the real axis for $\Psi_{m}$ and the Plancherel's Theorem, will provide the desired estimates for $\left\|\theta_{m}\right\|_{L^{2}(0, T)}$ and their dependence of the parameters $m, \varepsilon$ and $\alpha$.

\subsection{An entire function}

In this subsection we construct the Weierstrass product $P_{m}$ mentioned above and we study some of its properties. For every $m \in \mathbb{Z}^{*}$, we define the function

$$
P_{m}(z)=\prod_{\substack{n \in \mathbb{Z}^{*} \\ n \neq m}}\left(1+\frac{z i}{\bar{\lambda}_{n}}\right)\left(\frac{\bar{\lambda}_{n}}{\bar{\lambda}_{n}-\bar{\lambda}_{m}}\right) .
$$

Firstly, let us state the following technical result concerning the second part of the product $P_{m}$, whose proof will be given in the Appendix.

Lemma 3.1. There exists a constant $C>0$ such that, for all $\varepsilon \in(0,1)$ and $m \in \mathbb{Z}^{*}$, we have

$$
\prod_{\substack{n \in \mathbb{Z}^{*} \\ n \neq m}}\left|\frac{\lambda_{n}}{\lambda_{n}-\lambda_{m}}\right| \leq 16 \exp \left(C \varepsilon m^{2 \alpha}\right) .
$$

Now we pass to study the basic properties of the product $P_{m}$.

Proposition 3.2. Let $\alpha \in[0,1) \backslash\left\{\frac{1}{2}\right\}$ and $\varepsilon \in(0,1)$. For each $m \in \mathbb{Z}^{*}, P_{m}$ is an entire function of exponential type at most $L_{1}$, where

$$
L_{1}:=\left\{\begin{array}{l}
\max \left\{\frac{\sqrt{2} \pi}{2}, \frac{4 \varepsilon}{1-2 \alpha}\right\} \alpha \in\left[0, \frac{1}{2}\right) \\
\max \left\{\frac{\sqrt{2} \pi}{2}, \frac{8}{2 \alpha-1}\right\} \alpha \in\left(\frac{1}{2}, 1\right),
\end{array}\right.
$$

with the property that

$$
P_{m}\left(i \bar{\lambda}_{n}\right)=\delta_{\mathrm{mn}} \quad\left(n \in \mathbb{Z}^{*}\right)
$$

Remark 3.3. Note that Proposition 3.2 does not consider the case $\alpha=\frac{1}{2}$. In fact, if $\alpha=\frac{1}{2}$, the family of exponential functions $\left(\mathrm{e}^{\lambda_{n} t}\right)_{n \in \mathbb{Z}^{*}}$ is complete in $L^{2}(0, a)$, for any $a>0$. Indeed, since

$$
\sum_{n \in \mathbb{Z}^{*}} \frac{\Re\left(\lambda_{n}\right)}{1+\left|\lambda_{n}\right|^{2}}=\infty
$$

the completeness is a consequence of the Theorem Szász-Müntz [33]. Since this property remains true if we eliminate a finite number of elements, we deduce that $\left(\mathrm{e}^{\lambda_{n} t}\right)_{n \in \mathbb{Z}^{*}}$ is not minimal in $L^{2}(0, a)$ and there exists no biorthogonal sequence to it in $L^{2}(0, a)$. From the controllability point of view, it follows that (1.13) is not spectrally controllable if $\alpha=\frac{1}{2}$.

Proof of Proposition 3.2. By taking into account the estimate (3.2) from Lemma 3.1, we only have to study the function

$$
E_{m}(z)=\prod_{\substack{n \in \mathbb{Z}^{*} \\ n \neq m}}\left(1+\frac{z i}{\bar{\lambda}_{n}}\right)
$$


We have that

$$
\begin{aligned}
\left|E_{m}(z)\right| & =\left|1+\frac{z i}{\lambda_{m}}\right| \prod_{\substack{n \in \mathbb{N}^{*} \\
n \neq|m|}}\left|\left(1+\frac{z i}{\bar{\lambda}_{n}}\right)\left(1+\frac{z i}{\lambda_{n}}\right)\right| \leq\left(1+\frac{|z|}{\left|\lambda_{m}\right|}\right) \prod_{n=1}^{\infty} \frac{\left|\lambda_{n}\right|^{2}+2\left|\Re\left(\lambda_{n}\right)\right||z|+|z|^{2}}{\left|\lambda_{n}\right|^{2}} \\
& =\left(1+\frac{|z|}{\left|\lambda_{m}\right|}\right) \exp (\underbrace{\sum_{n=1}^{\left[N_{z}\right]} \ln \left(1+\frac{2\left|\Re\left(\lambda_{n}\right)\right||z|+|z|^{2}}{\left|\lambda_{n}\right|^{2}}\right)}_{S_{1}}+\underbrace{\sum_{n=\left[N_{z}\right]+1}^{\infty} \ln \left(1+\frac{2\left|\Re\left(\lambda_{n}\right)\right||z|+|z|^{2}}{\left|\lambda_{n}\right|^{2}}\right)}_{S_{2}}),
\end{aligned}
$$

where $N_{z}=\left(\frac{|z|}{2 \varepsilon}\right)^{\frac{1}{2 \alpha}}$. It follows that

$$
\begin{aligned}
S_{1} & \leq \sum_{n=1}^{\left[N_{z}\right]} \ln \left(1+\frac{2|z|^{2}}{\left|\lambda_{n}\right|^{2}}\right) \leq \int_{0}^{N_{z}} \ln \left(1+\frac{2|z|^{2}}{t^{2}+\varepsilon^{2} t^{4 \alpha}}\right) \mathrm{d} t \leq \int_{0}^{N_{z}} \ln \left(1+\frac{2|z|^{2}}{t^{2}}\right) \mathrm{d} t \\
& \leq \sqrt{2}|z| \int_{0}^{\infty} \ln \left(1+\frac{1}{t^{2}}\right) \mathrm{d} t=\frac{\sqrt{2} \pi}{2}|z| .
\end{aligned}
$$

Thus, we have that

$$
S_{1} \leq \frac{\sqrt{2} \pi}{2}|z|
$$

For $\alpha \in\left[0, \frac{1}{2}\right)$ we have that

$$
S_{2} \leq 4|z| \sum_{n=\left[N_{z}\right]+1}^{\infty} \frac{\varepsilon n^{2 \alpha}}{n^{2}+\varepsilon^{2} n^{4 \alpha}} \leq 4|z| \sum_{n=2}^{\infty} \varepsilon n^{2 \alpha-2} \leq \frac{4 \varepsilon}{1-2 \alpha}|z| .
$$

For $\alpha \in\left(\frac{1}{2}, 1\right)$ we define $\gamma_{\varepsilon}=\left(\frac{1}{\varepsilon}\right)^{\frac{1}{2 \alpha-1}}$ and we deduce that

$$
\begin{aligned}
S_{2} & \leq \sum_{n=\left[N_{z}\right]+1}^{\infty} \frac{4\left|\Re\left(\lambda_{n}\right)\right||z|}{\left|\lambda_{n}\right|^{2}}=4|z| \sum_{n=\left[N_{z}\right]+1}^{\infty} \frac{\varepsilon n^{2 \alpha}}{n^{2}+\varepsilon^{2} n^{4 \alpha}} \leq 4|z|\left(\sum_{n=1}^{\left[\gamma_{\varepsilon}\right]}+\sum_{n=\left[\gamma_{\varepsilon}\right]+1}^{\infty}\right) \frac{\varepsilon n^{2 \alpha}}{n^{2}+\varepsilon^{2} n^{4 \alpha}} \\
& \leq 4|z|\left(\sum_{n=1}^{\left[\gamma_{\varepsilon}\right]} \frac{\varepsilon}{n^{2-2 \alpha}}+\sum_{n=\left[\gamma_{\varepsilon}\right]+1}^{\infty} \frac{1}{\varepsilon n^{2 \alpha}}\right) \leq \frac{8}{2 \alpha-1}|z| .
\end{aligned}
$$

It follows that

$$
S_{2} \leq\left\{\begin{array}{l}
\frac{4 \varepsilon}{1-2 \alpha}|z| \alpha \in\left[0, \frac{1}{2}\right) \\
\frac{8}{2 \alpha-1}|z| \alpha \in\left(\frac{1}{2}, 1\right)
\end{array}\right.
$$

From (3.5) and (3.6) we deduce that $E_{m}(z)$ is an entire function of exponential type at most $L_{1}$ and the proof ends.

\subsection{Evaluation of $\boldsymbol{P}_{m}$ on the real axis}

This subsection is devoted to study the behavior of the entire function $P_{m}$ on the real axis. The main result will be presented in Proposition 3.6 below. Let us begin with the following two simple lemmas. We recall that, for $\alpha>\frac{1}{2}$, we have introduced the notation $\gamma_{\varepsilon}=\left(\frac{1}{\varepsilon}\right)^{\frac{1}{2 \alpha-1}}$.

Lemma 3.4. Let $\varepsilon \in(0,1)$ be fixed and $\alpha \in[0,1) \backslash\left\{\frac{1}{2}\right\}$. For any $x \geq 0$ there exists a unique $x_{\varepsilon} \geq 0$ such that $x^{2}=x_{\varepsilon}^{2}+\varepsilon^{2} x_{\varepsilon}^{4 \alpha}$. Moreover, if $\alpha \in\left[0, \frac{1}{2}\right)$ or $\alpha \in\left(\frac{1}{2}, 1\right)$ and $x \leq \gamma_{\varepsilon}$, then

$$
x_{\varepsilon} \leq x \leq \sqrt{2} x_{\varepsilon},
$$




$$
|x-| \lambda_{n}|| \geq \frac{\left|x_{\varepsilon}-n\right|}{\sqrt{2}} \quad\left(n \in \mathbb{N}^{*}, n \leq \gamma_{\varepsilon}\right) .
$$

Finally, if $\alpha \in\left(\frac{1}{2}, 1\right)$ and $x>\gamma_{\varepsilon}$, then

$$
\left|\lambda_{n}\right|-x \geq \frac{\varepsilon\left(n^{2 \alpha}-x_{\varepsilon}^{2 \alpha}\right)}{2 \sqrt{2}} \quad\left(n \in \mathbb{N}^{*}, n>\gamma_{\varepsilon}\right) .
$$

Proof. Let us first note that, for all $t \geq 0$, the equation

$$
r^{2}+\varepsilon^{2} r^{4 \alpha}=t
$$

has only one solution in $[0, \infty)$. Indeed, if we define the function $f:[0, \infty) \rightarrow \mathbb{R}, f(r)=r^{2}+\varepsilon^{2} r^{4 \alpha}-t$, it results that $f$ is increasing. Therefore equation (3.10) has at most one solution in $[0, \infty)$. On the other hand, we notice that $f(0)=-t$ and $\lim _{r \rightarrow \infty} f(r)=\infty$, from which we conclude that equation (3.10) has a unique solution in $[0, \infty)$. Concerning $(3.7)$, it is obviously that $x_{\varepsilon} \leq x$ and for the second part of the inequality we notice that

$$
x^{2}=x_{\varepsilon}^{2}+\varepsilon^{2} x_{\varepsilon}^{4 \alpha} \leq 2 x_{\varepsilon}^{2}
$$

for any $\alpha \in\left[0, \frac{1}{2}\right)$ or $\alpha \in\left(\frac{1}{2}, 1\right)$ and $x_{\varepsilon} \leq \gamma_{\varepsilon}$. Finally, taking into account that

$$
|x-| \lambda_{n}||=\left|\frac{x_{\varepsilon}^{2}-n^{2}+\varepsilon\left(x_{\varepsilon}^{4 \alpha}-n^{4 \alpha}\right)}{\sqrt{x_{\varepsilon}^{2}+\varepsilon^{2} x_{\varepsilon}^{4 \alpha}}+\sqrt{n^{2}+\varepsilon^{2} n^{4 \alpha}}}\right|,
$$

relations (3.8) and (3.9) follows immediately.

Lemma 3.5. The following inequalities hold

$$
\begin{gathered}
\frac{n^{4 \alpha-2}-x^{4 \alpha-2}}{n^{2}-x^{2}} \leq\left\{\begin{array}{l}
x^{4 \alpha-4} n \leq x \\
n^{4 \alpha-4} 0 \leq x \leq n
\end{array} \quad\left(\alpha \in\left(\frac{1}{2}, 1\right)\right),\right. \\
\frac{n^{4 \alpha-2}-x^{4 \alpha-2}}{n^{2}-x^{2}} \leq 0 \quad\left(\alpha \in\left[0, \frac{1}{2}\right)\right) .
\end{gathered}
$$

Proof. We notice that, when $x \geq n$, then

$$
\frac{n^{4 \alpha-2}-x^{4 \alpha-2}}{n^{2}-x^{2}}=x^{4 \alpha-4} \frac{\left(\frac{n}{x}\right)^{4 \alpha-2}-1}{\left(\frac{n}{x}\right)^{2}-1} \leq \begin{cases}0 & \alpha \in\left[0, \frac{1}{2}\right) \\ x^{4 \alpha-4} & \alpha \in\left(\frac{1}{2}, 1\right)\end{cases}
$$

The case $n \leq x$ is treated similarly.

The main result from this subsection is the following estimate of the function $P_{m}$ on the real axis.

Proposition 3.6. Let $\varepsilon \in(0,1)$ and $m \in \mathbb{Z}^{*}$. For each $\alpha \in[0,1) \backslash \frac{1}{2}$ there exist two positive constants $C$ and $\omega$, independent of $\varepsilon$ and $m$, such that the function $P_{m}$ defined by (3.1) verifies

$$
\left|P_{m}(x)\right| \leq C \exp \left[\omega\left(\varphi_{\varepsilon}(x)+\left|\Re\left(\lambda_{m}\right)\right|\right)\right] \quad(x \in \mathbb{R}),
$$

where

$$
\varphi_{\varepsilon}(x)= \begin{cases}\varepsilon|x|^{2 \alpha} & \text { if }\left(\alpha \in\left[0, \frac{1}{2}\right) \text { and } x \in \mathbb{R}\right) \quad \text { or } \quad\left(\alpha \in\left(\frac{1}{2}, 1\right) \text { and }|x| \leq \gamma_{\varepsilon}\right) \\ \left(\frac{|x|}{\varepsilon}\right)^{\frac{1}{2 \alpha}} & \text { if } \alpha \in\left(\frac{1}{2}, 1\right) \text { and }|x|>\gamma_{\varepsilon} .\end{cases}
$$


Proof. With the notations from Proposition 3.2 and by taking into account estimate (3.2) from Lemma 3.1, it follows that it is enough to evaluate $\left|E_{m}(x)\right|$. Moreover, since $P_{m}$ is a continuous function it is sufficient to consider $x \neq\left|\lambda_{n}\right|$ for all $n \in \mathbb{Z}^{*}$. In the sequel, $C$ denotes a generic constant which may change from one row to another but it is always independent of $\varepsilon$ and $m$.

To begin with, we evaluate $E_{m}$ on the real axis in the case $\left(\alpha \in\left[0, \frac{1}{2}\right)\right.$ and $\left.x \in \mathbb{R}\right)$ or $\left(\alpha \in\left(\frac{1}{2}, 1\right)\right.$ and $\left.|x| \leq \gamma_{\varepsilon}\right)$.

$$
\begin{aligned}
\left|E_{m}(x)\right|^{2} & =\left|\prod_{\substack{n \in \mathbb{Z}^{*} \\
n \neq m}}\left(1+\frac{x i}{\bar{\lambda}_{n}}\right)\right|^{2}=\left|1+\frac{x i}{\lambda_{m}}\right|^{2} \prod_{\substack{n=1 \\
n \neq m}}^{\infty}\left|\frac{\left|\lambda_{n}\right|^{2}+2 x i \Re\left(\lambda_{n}\right)-x^{2}}{\left|\lambda_{n}\right|^{2}}\right|^{2} \\
& =\frac{(x+m)^{2}+\varepsilon^{2} m^{4 \alpha}}{m^{2}+\varepsilon^{2} m^{4 \alpha}} \underbrace{\prod_{\substack{n=1 \\
n \neq m}}^{\infty} \frac{\left(\left|\lambda_{n}\right|^{2}-x^{2}\right)^{2}}{\left|\lambda_{n}\right|^{4}}}_{E_{m}^{1}(x)} \underbrace{\prod_{\substack{n=1 \\
n \neq m}}^{\infty} \frac{\left(\left|\lambda_{n}\right|^{2}-x^{2}\right)^{2}+4 x^{2}\left(\Re \lambda_{n}\right)^{2}}{\left(\left|\lambda_{n}\right|^{2}-x^{2}\right)^{2}}}_{E_{m}^{2}(x)} .
\end{aligned}
$$

We shall consider that $x \geq 0$. The opposite case can be treated in a similar way. Now, we evaluate $E_{m}^{1}(x)$ by using Lemma 3.4. We have that

$$
\begin{aligned}
\left|E_{m}^{1}(x)\right| & =\prod_{\substack{n=1 \\
n \neq m}}^{\infty}\left|\frac{\left|\lambda_{n}\right|^{2}-x^{2}}{\left|\lambda_{n}\right|^{2}}\right|^{2}=\prod_{\substack{n=1 \\
n \neq m}}^{\infty}\left|\frac{n^{2}+\varepsilon^{2} n^{4 \alpha}-x_{\varepsilon}^{2}-\varepsilon^{2} x_{\varepsilon}^{4 \alpha}}{n^{2}+\varepsilon^{2} n^{4 \alpha}}\right|^{2} \\
& =\left(\frac{m^{2}}{m^{2}-x_{\varepsilon}^{2}}\right)^{2} \prod_{n=1}^{\infty}\left|\frac{n^{2}-x_{\varepsilon}^{2}}{n^{2}}\right|^{2} \underbrace{\prod_{n=1}^{\infty}\left|1+\frac{\varepsilon^{2} x_{\varepsilon}^{2}}{1+\varepsilon^{2} n^{4 \alpha-2}} \frac{n^{4 \alpha-2}-x_{\varepsilon}^{4 \alpha-2}}{n^{2}-x_{\varepsilon}^{2}}\right|^{2}}_{\substack{n=1 \\
n \neq m}} \\
& =\left(\frac{m^{2}}{m^{2}-x_{\varepsilon}^{2}}\right)^{2}\left(\frac{\sin \pi x_{\varepsilon}}{\pi x_{\varepsilon}}\right)^{2} A_{m}^{1}(x) \leq \frac{m^{2}}{\left(m-x_{\varepsilon}\right)^{2}}\left(\frac{\sin \pi x_{\varepsilon}}{\pi x_{\varepsilon}}\right)^{2} A_{m}^{1}(x) .
\end{aligned}
$$

From Lemma 3.5 we deduce that, if $\alpha \in\left[0, \frac{1}{2}\right)$, the product $A_{m}^{1}(x)$ has the following property

$$
A_{m}^{1}(x) \leq 1 \text {. }
$$

On the other hand, if $\alpha \in\left(\frac{1}{2}, 1\right)$, from (3.11) we deduce that

$$
\begin{aligned}
A_{m}^{1}(x) & \leq \exp \left(2 \sum_{n=1}^{\left[x_{\varepsilon}\right]} \ln \left(1+\frac{\varepsilon^{2} x_{\varepsilon}^{4 \alpha-2}}{1+\varepsilon^{2} n^{4 \alpha-2}}\right)+2 \sum_{n=\left[x_{\varepsilon}\right]+1}^{\infty} \ln \left(1+\frac{x_{\varepsilon}^{2} \varepsilon^{2} n^{4 \alpha-4}}{1+\varepsilon^{2} n^{4 \alpha-2}}\right)\right) \\
& \leq \exp (2 \underbrace{\left[\sum_{n=1}^{\left[x_{\varepsilon}\right]} \frac{\varepsilon^{2} x_{\varepsilon}^{4 \alpha-2}}{1+\varepsilon^{2} n^{4 \alpha-2}}\right.}_{A_{m}^{11}(x)}+\underbrace{2 \sum_{n=\left[x_{\varepsilon}\right]+1}^{\infty} \frac{x_{\varepsilon}^{2} \varepsilon^{2} n^{4 \alpha-4}}{1+\varepsilon^{2} n^{4 \alpha-2}}}_{A_{m}^{12}(x)}) .
\end{aligned}
$$

Next, we proceed to evaluate the sums $A_{m}^{11}(x)$ and $A_{m}^{12}(x)$. Firstly, we have that

$$
A_{m}^{11}(x)=2 \sum_{n=1}^{\left[x_{\varepsilon}\right]} \frac{\varepsilon^{2} x_{\varepsilon}^{4 \alpha-2}}{1+\varepsilon^{2} n^{4 \alpha-2}} \leq 2 \int_{0}^{x_{\varepsilon}} \frac{\varepsilon^{2} x_{\varepsilon}^{4 \alpha-2}}{1+\varepsilon^{2} t^{4 \alpha-2}} \mathrm{~d} t \leq 2 \int_{0}^{x_{\varepsilon}} \varepsilon^{2} x_{\varepsilon}^{4 \alpha-2} \mathrm{~d} t \leq 2 \varepsilon^{2} x_{\varepsilon}^{4 \alpha-1} \leq 2 \varepsilon x_{\varepsilon}^{2 \alpha},
$$

and secondly we deduce that

$$
A_{m}^{12}(x)=\sum_{n=\left[x_{\varepsilon}\right]+1}^{\infty} \frac{2 x_{\varepsilon}^{2} \varepsilon^{2} n^{4 \alpha-4}}{1+\varepsilon^{2} n^{4 \alpha-2}} \leq \int_{x_{\varepsilon}}^{\infty} \frac{2 x_{\varepsilon}^{2} \varepsilon^{2} t^{4 \alpha-4}}{1+\varepsilon^{2} t^{4 \alpha-2}} \mathrm{~d} t=\int_{x_{\varepsilon}}^{\gamma_{\varepsilon}} \frac{2 x_{\varepsilon}^{2} \varepsilon^{2} t^{4 \alpha-4}}{1+\varepsilon^{2} t^{4 \alpha-2}} \mathrm{~d} t+\int_{\gamma_{\varepsilon}}^{\infty} \frac{2 x_{\varepsilon}^{2} \varepsilon^{2} t^{4 \alpha-4}}{1+\varepsilon^{2} t^{4 \alpha-2}} \mathrm{~d} t \leq 2 \varepsilon x_{\varepsilon}^{2 \alpha} .
$$


Consequently, we have proved that

$$
E_{m}^{1}(x) \leq \begin{cases}\frac{m^{2}}{\left(m-x_{\varepsilon}\right)^{2}}\left(\frac{\sin \pi x_{\varepsilon}}{\pi x_{\varepsilon}}\right)^{2} & \alpha \in\left[0, \frac{1}{2}\right), x \in \mathbb{R} \\ \frac{m^{2}}{\left(m-x_{\varepsilon}\right)^{2}}\left(\frac{\sin \pi x_{\varepsilon}}{\pi x_{\varepsilon}}\right)^{2} \exp \left(4 \varepsilon x^{2 \alpha}\right) & \alpha \in\left(\frac{1}{2}, 1\right), x \leq \gamma_{\varepsilon} .\end{cases}
$$

Nextly, we evaluate the product $E_{m}^{2}(x)$. In the following estimates we shall use the notation $n_{x}=\left[x_{\varepsilon}\right]$ and relations (3.7)-(3.8) from Lemma 3.4.

$$
\begin{aligned}
E_{m}^{2}(x)= & \underbrace{\frac{\left(\left|\lambda_{m}\right|^{2}-x^{2}\right)^{2}}{\left(\left|\lambda_{m}\right|^{2}-x^{2}\right)^{2}+4 x^{2} \Re^{2}\left(\lambda_{m}\right)}\left(1+\frac{4 x^{2} \Re^{2}\left(\lambda_{n_{x}}\right)}{\left(\left|\lambda_{n_{x}}\right|^{2}-x^{2}\right)^{2}}\right)\left(1+\frac{4 x^{2} \Re^{2}\left(\lambda_{n_{x}+1}\right)}{\left(\left|\lambda_{n_{x}+1}\right|^{2}-x^{2}\right)^{2}}\right)}_{E_{m}^{21}(x)} \\
& \prod_{n=1}^{n_{x}-1}\left(1+\frac{4 x^{2} \Re^{2}\left(\lambda_{n}\right)}{\left(\left|\lambda_{n}\right|^{2}-x^{2}\right)^{2}}\right) \prod_{n=n_{x}+2}^{\infty}\left(1+\frac{4 x^{2} \Re^{2}\left(\lambda_{n}\right)}{\left(\left|\lambda_{n}\right|^{2}-x^{2}\right)^{2}}\right) \\
\leq & E_{m}^{21}(x) \prod_{n=1}^{n_{x}-1}\left(1+\frac{4 \Re^{2}\left(\lambda_{n}\right)}{\left(\left|\lambda_{n}\right|-x\right)^{2}}\right) \prod_{n=n_{x}+2}^{\infty}\left(1+\frac{4 x^{2} \Re^{2}\left(\lambda_{n}\right)}{\left|\lambda_{n}\right|^{2}\left(\left|\lambda_{n}\right|-x\right)^{2}}\right) \\
= & E_{m}^{21}(x) \exp [\underbrace{\sum_{n=1}^{n_{x}-1} \ln \left(1+\frac{4 \Re^{2}\left(\lambda_{n}\right)}{\left(x-\left|\lambda_{n}\right|\right)^{2}}\right)}_{E_{m}^{22}(x)}+\underbrace{\sum_{n=n_{x}+2}^{\infty} \ln \left(1+\frac{4 x^{2} \Re^{2}\left(\lambda_{n}\right)}{\left|\lambda_{n}\right|^{2}\left(\left|\lambda_{n}\right|-x\right)^{2}}\right)}_{E_{m}^{23}(x)} .
\end{aligned}
$$

We estimate $E_{m}^{22}(x)$ by using Lemma 3.4 as follows

$$
E_{m}^{22}(x) \leq \sum_{n=1}^{n_{x}-1} \ln \left(1+\frac{8 \varepsilon^{2} n^{4 \alpha}}{\left(n_{x}-n\right)^{2}}\right) \leq 32 \alpha \int_{0}^{n_{x}} \frac{n_{x} \varepsilon^{2} t^{4 \alpha-1}}{\left(n_{x}-t\right)^{2}+8 \varepsilon^{2} t^{4 \alpha}} \mathrm{d} t .
$$

To bound from above the last integral we have to split the interval $\left(0, n_{x}\right)$ in three parts, by taking into account the following inequalities

$$
0 \leq \frac{n_{x}}{2} \leq n_{x}-\frac{\varepsilon n_{x}^{2 \alpha}}{1+\varepsilon n_{x}^{2 \alpha-1}} \leq n_{x}
$$

Thus, we have that

$$
\begin{aligned}
& \int_{0}^{n_{x}} \frac{n_{x} \varepsilon^{2} t^{4 \alpha-1}}{\left(n_{x}-t\right)^{2}+8 \varepsilon^{2} t^{4 \alpha}} \mathrm{d} t \leq \int_{0}^{\frac{n_{x}}{2}} \frac{\varepsilon^{2} n_{x} t^{4 \alpha-1}}{\left(n_{x}-t\right)^{2}} \mathrm{~d} t+\int_{\frac{n_{x}}{2}}^{n_{x}-\frac{\varepsilon n_{x}^{2 \alpha}}{1+\varepsilon n_{x}^{2}}} \frac{\varepsilon^{2} n_{x} t^{4 \alpha-1}}{\left(n_{x}-t\right)^{2}} \mathrm{~d} t+\int_{n_{x}-\frac{\varepsilon n_{x}^{2 \alpha}}{1+\varepsilon n_{x}^{2 \alpha-1}}}^{n_{x}} \frac{n_{x}}{t} \mathrm{~d} t \\
& \leq \int_{0}^{\frac{n_{x}}{2}} \frac{\varepsilon^{2} n_{x} t^{4 \alpha-1}}{\left(\frac{n_{x}}{2}\right)^{2}} \mathrm{~d} t+\int_{\frac{n_{x}}{2}}^{n_{x}-\frac{\varepsilon n_{x}^{2 \alpha}}{1+\varepsilon n_{x}^{2 \alpha-1}}} \frac{\varepsilon^{2} n_{x}^{4 \alpha}}{\left(n_{x}-t\right)^{2}} \mathrm{~d} t+n_{x} \ln \left(1+\varepsilon n_{x}^{2 \alpha-1}\right) \leq C \varepsilon n_{x}^{2 \alpha} .
\end{aligned}
$$

The last inequality takes place because $\varepsilon^{2} n_{x}^{4 \alpha-1} \leq \varepsilon n_{x}^{2 \alpha}$ if $\left(\alpha \in\left[0, \frac{1}{2}\right)\right.$ and $\left.x \in \mathbb{R}\right)$ or $\left(\alpha \in\left(\frac{1}{2}, 1\right)\right.$ and $\left.x \leq \gamma_{\varepsilon}\right)$. Now let us evaluate $E_{m}^{23}(x)$ by treating separately the following cases:

Case I. $\alpha \in\left[0, \frac{1}{2}\right)$ and $x \in \mathbb{R}$. Using relation (3.8) from Lemma 3.4 we obtain that

$$
\begin{aligned}
c E_{m}^{23}(x) & \leq \int_{x_{\varepsilon}+1}^{\infty} \ln \left(1+\frac{8 x^{2} \varepsilon^{2} t^{4 \alpha-2}}{\left(t-x_{\varepsilon}\right)^{2}}\right) \mathrm{d} t \leq 32 \int_{x_{\varepsilon}+1}^{\infty} \frac{x^{2} \varepsilon^{2} t^{4 \alpha-2}}{\left(t-x_{\varepsilon}\right)^{2}+x^{2} \varepsilon^{2} t^{4 \alpha-2}} \mathrm{~d} t \\
& =32\left(\int_{x_{\varepsilon}+1}^{2 x_{\varepsilon}}+\int_{2 x_{\varepsilon}}^{\infty}\right) \frac{x^{2} \varepsilon^{2} t^{4 \alpha-2}}{\left(t-x_{\varepsilon}\right)^{2}+x^{2} \varepsilon^{2} t^{4 \alpha-2}} \mathrm{~d} t \leq C \varepsilon x_{\varepsilon}^{2 \alpha} .
\end{aligned}
$$


Case II. $\alpha \in\left(\frac{1}{2}, 1\right)$ and $x \leq \gamma_{\varepsilon}$. Using the relations (3.8) and (3.9) from Lemma 3.4 we have that

$$
E_{m}^{23}(x) \leq \underbrace{\int_{x_{\varepsilon}}^{\gamma_{\varepsilon}} \ln \left(1+\frac{8 x^{2} \varepsilon^{2} t^{4 \alpha-2}}{\left(t-x_{\varepsilon}\right)^{2}}\right) \mathrm{d} t}_{I_{1}}+\underbrace{\int_{\gamma_{\varepsilon}}^{\infty} \ln \left(1+\frac{32 x^{2}}{\varepsilon^{2}\left(t^{2 \alpha}-x_{\varepsilon}^{2 \alpha}\right)^{2}}\right) \mathrm{d} t}_{I_{2}}
$$

To evaluate $I_{1}$ we integrate by parts and we take into account that there exists a constant $v \in(0,1)$ such that $x_{\varepsilon} \leq v \gamma_{\varepsilon}$. The existence of this constant allows us to separate the interval $\left(x_{\varepsilon}, \gamma_{\varepsilon}\right)$ as follows

$$
\begin{aligned}
I_{1} & \leq 4 x^{2} \varepsilon^{\frac{1}{2 \alpha-1}}+8 x^{2} \varepsilon^{2}\left(\int_{x_{\varepsilon}}^{\frac{1}{v} x_{\varepsilon}}+\int_{\frac{1}{v} x_{\varepsilon}}^{\gamma_{\varepsilon}}\right) \frac{t^{4 \alpha-2}}{\left(t-x_{\varepsilon}\right)^{2}+4 x^{2} \varepsilon^{2} t^{4 \alpha-2}} \mathrm{~d} t \\
& \leq 4 \varepsilon x^{2 \alpha}+\int_{x_{\varepsilon}}^{\frac{1}{v} x_{\varepsilon}} \frac{C x_{\varepsilon}^{4 \alpha} \varepsilon^{2}}{\left(t-x_{\varepsilon}\right)^{2}+x_{\varepsilon}^{4 \alpha} \varepsilon^{2}} \mathrm{~d} t+\int_{\frac{1}{v} x_{\varepsilon}}^{\gamma_{\varepsilon}} \frac{C x_{\varepsilon}^{2} \varepsilon^{2} t^{4 \alpha-4}}{1+x_{\varepsilon}^{2} \varepsilon^{2} t^{4 \alpha-4}} \mathrm{~d} t \\
& \leq 4 \varepsilon x^{2 \alpha}+\int_{0}^{\infty} \frac{C x_{\varepsilon}^{4 \alpha} \varepsilon^{2}}{t^{2}+x_{\varepsilon}^{4 \alpha} \varepsilon^{2}} \mathrm{~d} t+\int_{\frac{1}{v} x_{\varepsilon}}^{\gamma_{\varepsilon}} C x_{\varepsilon}^{2} \varepsilon^{2} t^{4 \alpha-4} \mathrm{~d} t \leq C \varepsilon x^{2 \alpha} .
\end{aligned}
$$

In order to estimate $I_{2}$ we remark that $t^{2 \alpha}-x_{\varepsilon}^{2 \alpha} \geq\left(1-v^{2 \alpha}\right) t^{2 \alpha}$. It follows that

$$
I_{2} \leq \int_{\gamma_{\varepsilon}}^{\infty} \ln \left(1+\frac{32 x^{2}}{\left(1-v^{2 \alpha}\right)^{2} \varepsilon^{2} t^{4 \alpha}}\right) \mathrm{d} t \leq \int_{\gamma_{\varepsilon}}^{\infty} \frac{32 x^{2}}{\left(1-v^{2 \alpha}\right)^{2} \varepsilon^{2} t^{4 \alpha}} \mathrm{d} t \leq \frac{32}{(4 \alpha-1)\left(1-v^{2 \alpha}\right)^{2}} \varepsilon x^{2 \alpha} .
$$

Thus, we have that

$$
E_{m}^{2}(x) \leq E_{m}^{21}(x) \exp \left(C \varepsilon|x|^{2 \alpha}\right) .
$$

Note that, for any $m \in \mathbb{Z}^{*}$, there exists a positive constant $\widetilde{C}$, independent of $m$ and $\varepsilon$, such that

$$
\frac{(x+m)^{2}+\varepsilon^{2} m^{4 \alpha}}{m^{2}+\varepsilon^{2} m^{4 \alpha}} \frac{m^{2}}{\left(m-x_{\varepsilon}\right)^{2}}\left(\frac{\sin \pi x_{\varepsilon}}{\pi x_{\varepsilon}}\right)^{2}\left(1+\frac{4 x^{2} \Re^{2}\left(\lambda_{n_{x}}\right)}{\left(\left|\lambda_{n_{x}}\right|^{2}-x^{2}\right)^{2}}\right)\left(1+\frac{4 x^{2} \Re^{2}\left(\lambda_{n_{x}+1}\right)}{\left(\left|\lambda_{n_{x}+1}\right|^{2}-x^{2}\right)^{2}}\right) \leq \widetilde{C} .
$$

Indeed, the terms $\frac{m^{2}}{\left(m-x_{\varepsilon}\right)^{2}}, 1+\frac{4 x^{2} \Re^{2}\left(\lambda_{n_{x}}\right)}{\left(\left|\lambda_{n_{x}}\right|^{2}-x^{2}\right)^{2}}$ and $1+\frac{4 x^{2} \Re^{2}\left(\lambda_{n_{x}+1}\right)}{\left(\left|\lambda_{n_{x}+1}\right|^{2}-x^{2}\right)^{2}}$ explodes as $x$ tends to $m, \lambda_{n_{x}}$ and $\lambda_{n_{x}+1}$ respectively, but not simultaneously. This allows us to couple them with the sine function $\left(\frac{\sin \pi x_{\varepsilon}}{\pi x_{\varepsilon}}\right)^{2}$ in order to obtain a bounded function. On the other hand, when $x$ tends to infinity we couple the first two terms so that we obtain once again a bounded function.

Consequently, from (3.15), (3.17) and (3.18) it follows that, for every $\alpha \in[0,1) \backslash\left\{\frac{1}{2}\right\}$ there exists an absolute positive constant $C$, such that for any $\left(x \in \mathbb{R}\right.$ if $\left.\alpha \in\left[0, \frac{1}{2}\right)\right)$ or $\left(|x| \leq \gamma_{\varepsilon}\right.$ if $\left.\alpha \in\left(\frac{1}{2}, 1\right)\right)$, we have that

$$
\left|E_{m}(x)\right| \leq \exp \left(C \varepsilon x^{2 \alpha}\right) .
$$

To conclude the proof it remains to evaluate the product $E_{m}(x)$ in the case $\alpha \in\left(\frac{1}{2}, 1\right)$ and $|x|>\gamma_{\varepsilon}$. Note that

$$
\begin{array}{r}
\left|E_{m}(x)\right|^{2}=\left|\prod_{\substack{n \in \mathbb{Z}^{*} \\
n \neq m}}\left(1+\frac{x i}{\bar{\lambda}_{n}}\right)\right|^{2}=\left|1+\frac{x i}{\lambda_{m}}\right|^{2}\left|\prod_{\substack{n=1 \\
n \neq m}}^{\infty}\left(1+\frac{x i}{\lambda_{n}}\right)\left(1+\frac{x i}{\bar{\lambda}_{n}}\right)\right|^{2} \\
\leq \frac{(m+x)^{2}+\varepsilon^{2} m^{4 \alpha}}{m^{2}+\varepsilon^{2} m^{4 \alpha}} \exp [\underbrace{\sum_{n=1}^{\left[\eta_{\varepsilon}(x)\right]} \ln \left(1+\frac{x^{4}+4 x^{2} \Re^{2}\left(\lambda_{n}\right)}{\left|\lambda_{n}\right|^{4}}\right)}_{S_{m}^{1}(x)}+\underbrace{\sum_{n=\left[\eta_{\varepsilon}(x)\right]+1}^{\infty} \ln \left(1+\frac{x^{4}+4 x^{2} \Re^{2}\left(\lambda_{n}\right)}{\left|\lambda_{n}\right|^{4}}\right)}_{S_{m}^{2}(x)},
\end{array}
$$


where $\eta_{\varepsilon}(x)=\left(\frac{x}{\sqrt{2} \varepsilon}\right)^{\frac{1}{2 \alpha}}$. Now, we evaluate the above sum as follows.

$$
\begin{aligned}
S_{m}^{1}(x) \leq & \sum_{n=1}^{\left[\eta_{\varepsilon}(x)\right]} \ln \left(1+\frac{x^{4}}{\left|\lambda_{n}\right|^{4}}\right) \leq \int_{0}^{\eta_{\varepsilon}(x)} \ln \left(1+\frac{x^{4}}{\left(t^{2}+\varepsilon^{2} t^{4 \alpha}\right)^{2}}\right) \mathrm{d} t=\int_{0}^{\gamma_{\varepsilon}} \ln \left(1+\frac{x^{4}}{t^{4}}\right) \mathrm{d} t \\
& +\int_{\gamma_{\varepsilon}}^{\eta_{\varepsilon}(x)} \ln \left(1+\frac{x^{4}}{\varepsilon^{4} t^{8 \alpha}}\right) \mathrm{d} t C\left(\frac{x}{\varepsilon}\right)^{\frac{1}{2 \alpha}}, \\
S_{m}^{2}(x) \leq & \sum_{n=\left[\eta_{\varepsilon}(x)\right]+1}^{\infty} \frac{x^{4}+4 x^{2} \Re^{2}\left(\lambda_{n}\right)}{\left|\lambda_{n}\right|^{4}} \leq \sum_{n=\left[\eta_{\varepsilon}(x)\right]+1}^{\infty} \frac{8 x^{2} \Re^{2}\left(\lambda_{n}\right)}{\left|\lambda_{n}\right|^{4}}=\sum_{n=\left[\eta_{\varepsilon}(x)\right]+1}^{\infty} \frac{8 x^{2} \varepsilon^{2} n^{4 \alpha}}{\left(n^{2}+\varepsilon^{2} n^{4 \alpha}\right)^{2}} \\
\leq & \sum_{n=\left[\eta_{\varepsilon}(x)\right]+1}^{\infty} \frac{8 x^{2}}{\varepsilon^{2} n^{4 \alpha}} \leq \int_{\eta_{\varepsilon}(x)}^{\infty} \frac{8 x^{2}}{\varepsilon^{2}} t^{-4 \alpha} \mathrm{d} t \leq C\left(\frac{x}{\varepsilon}\right)^{\frac{1}{2 \alpha}} .
\end{aligned}
$$

Thus, for $\alpha \in\left(\frac{1}{2}, 1\right)$ and $|x|>\gamma_{\varepsilon}$, we have proved that

$$
\left|E_{m}(x)\right| \leq \exp \left(C\left(\frac{|x|}{\varepsilon}\right)^{\frac{1}{2 \alpha}}\right) .
$$

Now, by taking into account (3.19) and (3.20) the proof of the Proposition ends.

\subsection{A multiplier}

In this subsection we construct a function, called multiplier, used to compensate the grow of the product $P_{m}$ on the real axis given in Proposition 3.6.

Let $\varphi:[0, \infty) \rightarrow[0, \infty)$ be a continuous, increasing and onto function. We define the real sequence $\left(a_{n}\right)_{n \geq 1}$ by

$$
\varphi\left(e a_{n}\right)=n \quad(n \geq 1)
$$

and we suppose that the following properties hold:

(I1) $\sum_{n \geq 1} \frac{1}{a_{n}} \leq L_{2}<\infty$

(I2) $\sum_{n=n_{m}}^{\infty} \frac{1}{a_{n}^{2}} \leq D \frac{1+\left|\Re\left(\lambda_{m}\right)\right|}{\left|\lambda_{m}\right|^{2}}$,

where $L_{2}$ and $D$ are two positive constants and

$$
n_{m}=\left[\varphi\left(e\left|\lambda_{m}\right|\right)\right]+1 \quad(m \geq 1) .
$$

We have the following result.

Lemma 3.7. Let $x \geq a_{n_{m}}$ and define $n_{x}:=[\varphi(e x)]$. Then

$$
\sum_{j=n_{m}}^{n_{x}} \ln \left(\frac{a_{j}}{x}\right)=-\int_{a_{n_{m}}}^{x} \frac{A(u)-n_{m}+1}{u} \mathrm{~d} u,
$$

where $A(u)=\#\left\{a_{n} \leq u\right\}=\left[\varphi_{\varepsilon}(e u)\right]$. 
Proof. We have that

$$
\begin{aligned}
& -\int_{a_{n_{m}}}^{x} \frac{A(u)}{u} \mathrm{~d} u=-\sum_{j=n_{m}}^{n_{x}-1} \int_{a_{j}}^{a_{j+1}} \frac{A(u)}{u} \mathrm{~d} u-\int_{a_{n_{x}}}^{x} \frac{A(u)}{u} \mathrm{~d} u=-\sum_{j=n_{m}}^{n_{x}-1} \int_{a_{j}}^{a_{j+1}} \frac{j}{u} \mathrm{~d} u-\int_{a_{n_{x}}}^{x} \frac{n_{x}}{u} \mathrm{~d} u \\
& =\ln \left(\prod_{j=n_{m}}^{n_{x}-1} \frac{a_{j}^{j}}{a_{j+1}^{j}} \frac{a_{n_{x}}^{n_{x}}}{x^{n_{x}}}\right)=\ln \left(\frac{a_{n_{m}}^{n_{m}-1}}{x^{n_{m}-1}} \prod_{j=n_{m}}^{n_{x}} \frac{a_{j}}{x}\right)=-\int_{a_{n_{m}}}^{x} \frac{n_{m}-1}{u} \mathrm{~d} u+\sum_{j=n_{m}}^{n_{x}} \ln \left(\frac{a_{j}}{x}\right),
\end{aligned}
$$

which completes the proof of the lemma.

Now we can construct a multiplier function.

Theorem 3.8. Let $\varphi:[0, \infty) \rightarrow[0, \infty)$ be a continuous, increasing, onto function such that the sequence $\left(a_{n}\right)_{n \geq 1}$ defined by (3.21) verifies (I1) and (I2). For each $m \geq 1$ there exists $M_{m}: \mathbb{C} \rightarrow \mathbb{C}$ with the following properties:

1. $M_{m}$ is an entire function of exponential type $L_{2}$

2. $\left|M_{m}(x)\right| \leq \exp \left(-\varphi(|x|)+\varphi\left(e\left|\lambda_{m}\right|\right)+1\right)$ for all $x \in \mathbb{R}$

3. $\left|M_{m}\left(i \bar{\lambda}_{m}\right)\right| \geq \exp \left(-\frac{D}{6}\left(1+\left|\Re\left(\lambda_{m}\right)\right|\right)\right)$.

Proof. By adapting an idea from [19], we define the function $M_{m}: \mathbb{C} \rightarrow \mathbb{C}$ as follows

$$
M_{m}(z)=\prod_{n=n_{m}}^{\infty} \frac{\sin \left(\frac{z}{a_{n}}\right)}{\frac{z}{a_{n}}}
$$

where the sequence $\left(a_{n}\right)_{n \geq 1}$ is given by (3.21) and $n_{m}$ is defined in (3.22).

$M_{m}$ is an entire function of exponential type. Indeed, this is a consequence of property (I1) of the sequence $\left(a_{n}\right)_{n \geq 1}$ and the following estimate which holds for each $N>n_{m}$,

$$
\prod_{n=n_{m}}^{N}\left|\frac{\sin \left(\frac{z}{a_{n}}\right)}{\frac{z}{a_{n}}}\right| \leq \prod_{n=n_{m}}^{N} \mathrm{e}^{\left|\frac{z}{a_{n}}\right|}=\mathrm{e}^{|z| \sum_{n=n_{m}}^{N} \frac{1}{a_{n}}} \leq \mathrm{e}^{L_{2}|z|}
$$

To prove the second property of $M_{m}$ we need to analyze the following two cases:

Case 1. $x \leq e a_{n_{m}}$. We deduce that $\varphi(x) \leq \varphi\left(e a_{n_{m}}\right)=n_{m} \leq \varphi\left(e\left|\lambda_{m}\right|\right)+1$ and consequently

$$
\left|M_{m}(x)\right|=\prod_{n=n_{m}}^{\infty}\left|\frac{\sin \left(\frac{x}{a_{n}}\right)}{\frac{x}{a_{n}}}\right| \leq 1 \leq \exp \left(\varphi\left(e\left|\lambda_{m}\right|\right)-\varphi(x)+1\right) .
$$

Case 2. $e a_{n_{m}}<x$. From Lemma 3.7 we deduce that

$$
\left|M_{m}(x)\right|=\prod_{n=n_{m}}^{\infty}\left|\frac{\sin \left(\frac{x}{a_{n}}\right)}{\frac{x}{a_{n}}}\right| \leq \prod_{n=n_{m}}^{n_{x}}\left|\frac{a_{n}}{x}\right|=\exp \left(\sum_{n=n_{m}}^{n_{x}} \ln \left(\frac{a_{n}}{x}\right)\right)=\exp \left(-\int_{a_{n_{m}}}^{x} \frac{A(u)-n_{m}+1}{u} \mathrm{~d} u\right) .
$$

Since $a_{n_{m}}<\frac{x}{e}$, it follows that

$$
\left|M_{m}(x)\right| \leq \exp \left(-\int_{\frac{x}{e}}^{x} \frac{A(u)-n_{m}+1}{u} \mathrm{~d} u\right) \leq \exp \left(-\int_{\frac{x}{e}}^{x} \frac{\varphi(x)-1-n_{m}+1}{u} \mathrm{~d} u\right)=\exp \left(-\varphi(x)+n_{m}\right) .
$$

Since $n_{m}=\left[\varphi\left(e\left|\lambda_{m}\right|\right)\right]+1 \leq \varphi\left(e\left|\lambda_{m}\right|\right)+1$, the second property of $M_{m}$ is proved. 
To prove the third property of $M_{m}$, note that

$$
a_{n_{m}}=\frac{1}{e} \varphi^{-1}\left(n_{m}\right) \geq \frac{1}{e} \varphi^{-1}\left(\varphi\left(e\left|\lambda_{m}\right|\right)\right)=\left|\lambda_{m}\right|
$$

and consequently $\left|\frac{\lambda_{m}}{a_{n}}\right| \leq 1$ for each $n \geq n_{m}$. It follows that

$$
\begin{aligned}
\left|M_{m}\left(i \bar{\lambda}_{m}\right)\right| & =\prod_{n=n_{m}}^{\infty}\left|\frac{\sin \left(\frac{i \bar{\lambda}_{m}}{a_{n}}\right)}{\frac{i \bar{\lambda}_{m}}{a_{n}}}\right| \geq \prod_{n=n_{m}}^{\infty} \frac{\sin \left|\frac{\bar{\lambda}_{m}}{a_{n}}\right|}{\left|\frac{\bar{\lambda}_{m}}{a_{n}}\right|} \geq \prod_{n=n_{m}}^{\infty}\left|1-\frac{1}{6} \frac{\left|\bar{\lambda}_{m}\right|^{2}}{a_{n}^{2}}\right| \\
& =\exp \left(\sum_{n=n_{m}}^{\infty} \ln \left(1-\frac{1}{6} \frac{\left|\lambda_{m}\right|^{2}}{a_{n}^{2}}\right)\right) \geq \exp \left(-\frac{\left|\lambda_{m}\right|^{2}}{6} \sum_{n=n_{m}}^{\infty} \frac{1}{a_{n}^{2}}\right)
\end{aligned}
$$

By using property (I2) of the sequence $\left(a_{n}\right)_{n \geq 1}$, we deduce that the third property of $M_{m}$ also holds and the proof of the theorem ends.

Proposition 3.9. For $\alpha \in(0,1) \backslash\left\{\frac{1}{2}\right\}$ and $\varepsilon \in(0,1)$, let $\varphi_{\varepsilon}:[0, \infty) \rightarrow[0, \infty)$ be the function defined by (3.14). For each $m \geq 1$ there exists $M_{m}: \mathbb{C} \rightarrow \mathbb{C}$ with the following properties:

1. $M_{m}$ is an entire function of exponential type $L_{2}$

2. $\left|M_{m}(x)\right| \leq \exp \left(-\varphi_{\varepsilon}(x)+2 \mathrm{e}^{2}\left|\Re\left(\lambda_{m}\right)\right|+1\right)$ for all $x \in \mathbb{R}$

3. $\left.\left|M_{m}\left(i \bar{\lambda}_{m}\right)\right| \geq \exp \left(-D\left(1+\left|\Re\left(\lambda_{m}\right)\right|\right)\right)\right)$,

where $L_{2}$ and $D$ are positive constants independent of $m$ and $\varepsilon$.

Proof. The existence of the function $M_{m}$ follows from Theorem 3.8 if we prove that the function $\varphi_{\varepsilon}$ verifies the hypothesis from Theorem 3.8 and

$$
\varphi_{\varepsilon}\left(e\left|\lambda_{m}\right|\right) \leq 2 \mathrm{e}^{2}\left|\Re\left(\lambda_{m}\right)\right| \quad(m \geq 1) .
$$

From (3.14) we deduce immediately that $\varphi_{\varepsilon}:(0, \infty) \rightarrow(0, \infty)$ is continues, increasing, onto and (3.25) is verified. Moreover, the sequence $\left(a_{n}\right)_{n \geq 1}$ defined by $a_{n}=\frac{1}{e} \varphi^{-1}(n)$ verifies the following properties.

- For $\alpha \in\left(0, \frac{1}{2}\right)$ we have that

$$
\frac{1}{e} \sum_{n \geq 1} \frac{1}{a_{n}}=\varepsilon^{\frac{1}{2 \alpha}}+\varepsilon^{\frac{1}{2 \alpha}} \sum_{n \geq 2}\left(\frac{1}{n}\right)^{\frac{1}{2 \alpha}} \leq \frac{4 \alpha+1}{2 \alpha} \varepsilon^{\frac{1}{2 \alpha}} .
$$

- For $\alpha \in\left(\frac{1}{2}, 1\right)$ we have that

$$
\frac{1}{e} \sum_{n \geq 1} \frac{1}{a_{n}}=\sum_{n \geq 1} \frac{1}{\varphi_{\varepsilon}^{-1}(n)} \leq \int_{0}^{\infty} \frac{1}{\varphi_{\varepsilon}^{-1}(s)} d s=\int_{0}^{\gamma_{\varepsilon}}\left(\frac{\varepsilon}{s}\right)^{\frac{1}{2 \alpha}} d s+\int_{\gamma_{\varepsilon}}^{\infty} \frac{1}{\varepsilon s^{2 \alpha}} d s=\frac{2 \alpha+1}{2 \alpha-1} .
$$

By taking $L_{2}=\frac{4 \alpha+1}{2 \alpha} \varepsilon^{\frac{1}{2 \alpha}} e$ for $\alpha \in\left(0, \frac{1}{2}\right)$ and $L_{2}=\frac{2 \alpha+1}{2 \alpha-1} e$ for $\alpha \in\left(\frac{1}{2}, 1\right)$ it follows that hypothesis $(I 1)$ is verified.

On the other hand we have that

- For $\alpha \in\left(0, \frac{1}{2}\right)$ we have that

$$
\frac{1}{\mathrm{e}^{2}} \sum_{n=n_{m}}^{\infty} \frac{1}{a_{n}^{2}}=\sum_{n=n_{m}}^{\infty}\left(\frac{\varepsilon}{n}\right)^{\frac{1}{\alpha}} \leq \int_{\varphi_{\varepsilon}\left(e\left|\lambda_{m}\right|\right)}^{\infty}\left(\frac{\varepsilon}{s}\right)^{\frac{1}{\alpha}} \mathrm{d} s \leq \varepsilon\left|\lambda_{m}\right|^{2 \alpha-2} \leq \frac{1}{\left|\lambda_{m}\right|^{2}}\left(1+2^{\alpha}\left|\Re\left(\lambda_{m}\right)\right|\right) .
$$


- For $\alpha \in\left(\frac{1}{2}, 1\right)$ it follows that

$$
\frac{1}{\mathrm{e}^{2}} \sum_{n=n_{m}}^{\infty} \frac{1}{a_{n}^{2}}=\sum_{n=n_{m}}^{\infty} \frac{1}{\left(\varphi_{\varepsilon}^{-1}(n)\right)^{2}} \leq \frac{1}{\left(\varphi_{\varepsilon}^{-1}\left(n_{m}\right)\right)^{2}}+\int_{n_{m}}^{\infty} \frac{1}{\left(\varphi_{\varepsilon}^{-1}(s)\right)^{2}} \mathrm{~d} s
$$

Since $n_{m} \geq \varphi_{\varepsilon}\left(e\left|\lambda_{m}\right|\right)$ it results that

$$
\frac{1}{\left(\varphi_{\varepsilon}^{-1}\left(n_{m}\right)\right)^{2}} \leq \frac{1}{\mathrm{e}^{2}\left|\lambda_{m}\right|^{2}} \quad\left(m \in \mathbb{N}^{*}\right)
$$

On the other hand, if $e\left|\lambda_{m}\right| \leq \gamma_{\varepsilon}$ we have that

$$
\begin{aligned}
\int_{\varphi_{\varepsilon}\left(e\left|\lambda_{m}\right|\right)}^{\infty} \frac{1}{\left(\varphi_{\varepsilon}^{-1}(s)\right)^{2}} \mathrm{~d} s & =\int_{\varphi_{\varepsilon}\left(e\left|\lambda_{m}\right|\right)}^{\gamma_{\varepsilon}}\left(\frac{\varepsilon}{s}\right)^{\frac{1}{\alpha}} \mathrm{d} s+\int_{\gamma_{\varepsilon}}^{\infty} \frac{1}{\varepsilon^{2} s^{4 \alpha}} \mathrm{d} s \leq \\
& \leq \frac{\alpha}{1-\alpha} \varepsilon^{\frac{1}{\alpha}}\left(\varepsilon\left(e\left|\lambda_{m}\right|\right)^{2 \alpha}\right)^{\frac{\alpha-1}{\alpha}}+\frac{1}{4 \alpha-1} \frac{1}{\varepsilon^{2}} \gamma_{\varepsilon}^{1-4 \alpha} \leq \frac{4 \alpha}{1-\alpha} \frac{1}{\left|\lambda_{m}\right|^{2}}\left|\Re\left(\lambda_{m}\right)\right|
\end{aligned}
$$

and if $e\left|\lambda_{m}\right|>\gamma_{\varepsilon}$

$$
\int_{\varphi_{\varepsilon}\left(e\left|\lambda_{m}\right|\right)}^{\infty} \frac{1}{\left(\varphi_{\varepsilon}^{-1}(s)\right)^{2}} \mathrm{~d} s=\int_{\varphi_{\varepsilon}\left(e\left|\lambda_{m}\right|\right)}^{\infty} \frac{1}{\varepsilon^{2} s^{4 \alpha}} \mathrm{d} s \leq \frac{1}{4 \alpha-1} \mathrm{e}^{\frac{1-4 \alpha}{2 \alpha}} \frac{1}{\varepsilon^{2}}\left(\frac{\left|\lambda_{m}\right|}{\varepsilon}\right)^{\frac{1-4 \alpha}{2 \alpha}} \leq \frac{4 \mathrm{e}^{2}}{4 \alpha-1} \frac{1}{\left|\lambda_{m}\right|^{2}}\left|\Re\left(\lambda_{m}\right)\right| .
$$

By taking $D=2^{\alpha} \mathrm{e}^{2}$ for $\alpha \in\left(0, \frac{1}{2}\right)$ and $D=\frac{4 \alpha}{1-\alpha} \mathrm{e}^{2}$ for $\alpha \in\left(\frac{1}{2}, 1\right)$ it follows that hypothesis (I2) is verified and the proof of the proposition finishes.

\subsection{Two biorthogonal sequences}

Now we have all the ingredients needed to construct a biorthogonal sequence $\left(\theta_{m}\right)_{m \in \mathbb{Z}^{*}}$ to the family $\left(\mathrm{e}^{\lambda_{n} t}\right)_{n \in \mathbb{Z}^{*}}$, by using the method presented in the section's introduction.

Theorem 3.10. Let $\varepsilon \in(0,1)$. There exist $\widetilde{T}>0$ independent of $\varepsilon$ and a biorthogonal sequence $\left(\theta_{m}\right)_{m \in \mathbb{Z}^{*}}$ to the family $\left(\mathrm{e}^{\lambda_{n} t}\right)_{n \in \mathbb{Z}^{*}}$ in $L^{2}\left(-\frac{\widetilde{T}}{2}, \frac{\widetilde{T}}{2}\right)$, with the following property

$$
\left\|\theta_{m}\right\|_{L^{2}\left(-\frac{\tilde{T}}{2}, \frac{\tilde{T}}{2}\right)} \leq C \exp \left(\beta\left|\Re\left(\lambda_{m}\right)\right|\right) \quad\left(m \in \mathbb{Z}^{*}\right)
$$

where $C$ and $\beta$ are positive constants independent of $m$ and $\varepsilon$.

Proof. If $\alpha \neq 0$, for each $m \in \mathbb{Z}^{*}$, let $P_{m}$ and $M_{|m|}$ be the functions from Propositions 3.2 and 3.9 , respectively. We define the function

$$
\Psi_{m}(z)=P_{m}(z)\left(\frac{M_{|m|}(z)}{M_{|m|}\left(i \bar{\lambda}_{m}\right)}\right)^{\omega} \frac{\sin \left(\delta\left(z-i \bar{\lambda}_{m}\right)\right)}{\delta\left(z-i \bar{\lambda}_{m}\right)}
$$

where $\delta>0$ is an arbitrary constant and $\omega$ is the constant from Proposition 3.6. Let

$$
\theta_{m}(t)=\frac{1}{2 \pi} \int_{\mathbb{R}} \Psi_{m}(x) \mathrm{e}^{i x t} \mathrm{~d} x .
$$

From Propositions 3.2 and 3.9 we deduce that there exists $\widetilde{T}=2\left(L_{1}+\omega L_{2}+\delta\right)$, independent of $\varepsilon$, such that $\Psi_{m}$ is an entire function of exponential type $\frac{\widetilde{T}}{2}$. Moreover, from the estimate of the function $P_{m}$ on the real axis 
given by Proposition 3.6 and the properties of the function $M_{|m|}$ from Proposition 3.9, we obtain that

$$
\begin{aligned}
\int_{\mathbb{R}}\left|\Psi_{m}(x)\right|^{2} \mathrm{~d} x & \leq C \mathrm{e}^{2 \omega(1+D)} \exp \left(2 \omega\left(1+2 \mathrm{e}^{2}+D\right)\left|\Re\left(\lambda_{m}\right)\right|\right) \int_{\mathbb{R}}\left|\frac{\sin \left(\delta\left(x-i \lambda_{m}\right)\right)}{\delta\left(x-i \lambda_{m}\right)}\right|^{2} \mathrm{~d} x \\
& \leq \frac{C}{\delta} \exp \left(2\left(\omega+2 \omega \mathrm{e}^{2}+\omega D+\delta\right)\left|\Re\left(\lambda_{m}\right)\right|\right) \int_{\mathbb{R}}\left|\frac{\sin t}{t}\right|^{2} \mathrm{~d} t \leq C \exp \left(2 \beta\left|\Re\left(\lambda_{m}\right)\right|\right),
\end{aligned}
$$

where $\beta$ is any number greater that $\omega+2 \omega \mathrm{e}^{2}+\omega D+\delta$.

Now, by taking into account the properties of $\Psi_{m}$ and by applying the Paley-Wiener Theorem, [35], Theorem 18, Section 2.4, we deduce that $\theta_{m} \in L^{2}\left(-\frac{\widetilde{T}}{2}, \frac{\widetilde{T}}{2}\right)$. Moreover, from the inverse Fourier transform property we obtained that $\left(\theta_{m}\right)_{m \in \mathbb{Z}^{*}}$ is a biorthogonal sequence to $\left(\mathrm{e}^{\lambda_{n} t}\right)_{n \in \mathbb{Z}^{*}}$ in $L^{2}\left(-\frac{\widetilde{T}}{2}, \frac{\widetilde{T}}{2}\right)$. Finally, from Plancherel's Theorem we deduce that (3.27) holds.

If $\alpha=0$, we take

$$
\Psi_{m}(z)=P_{m}(z) \frac{\sin \left(\delta\left(z-i \bar{\lambda}_{m}\right)\right)}{\delta\left(z-i \bar{\lambda}_{m}\right)},
$$

where $\delta>0$ is an arbitrary constant. The same argument as before allows us to end the proof of the theorem.

The following result gives the existence of a new biorthogonal sequence with better norm properties than the one from Theorem 3.10 .

Theorem 3.11. Let $\varepsilon \in(0,1)$. There exist $T_{0}>0$ independent of $\varepsilon$ and a biorthogonal sequence $\left(\zeta_{m}\right)_{m \in \mathbb{Z}^{*}}$ to the family $\left(\mathrm{e}^{\lambda_{n} t}\right)_{n \in \mathbb{Z}^{*}}$ in $L^{2}\left(-\frac{T_{0}}{2}, \frac{T_{0}}{2}\right)$, such that, for any finite sequence $\left(c_{m}\right)_{m \in \mathbb{Z}^{*}}$, we have

$$
\int_{-\frac{T_{0}}{2}}^{\frac{T_{0}}{2}}\left|\sum_{m \in \mathbb{Z}^{*}} c_{m} \zeta_{m}(t)\right|^{2} \mathrm{~d} t \leq C\left(T_{0}\right) \sum_{m \in \mathbb{Z}^{*}}\left|c_{m}\right|^{2} \mathrm{e}^{2 \beta\left|\Re\left(\lambda_{m}\right)\right|},
$$

where $\beta$ is the same as in Theorem 3.10 and $C\left(T_{0}\right)$ is a constant depending only of $T_{0}$.

Proof. Since it is similar to that of Theorem 3.4 from [27], we only give the main ideas. Let $\left(\theta_{m}\right)_{m \in \mathbb{Z}^{*}} \subset$ $L^{2}\left(-\frac{\widetilde{T}}{2}, \frac{\widetilde{T}}{2}\right)$ be the biorthogonal sequence from Theorem 3.10. For any $a>0$ define $k_{a}=\frac{\sqrt{2 \pi}}{a^{2}}\left(\chi_{a} * \chi_{a}\right)$, where $\chi_{a}$ represents the characteristic function $\chi_{[-a / 2, a / 2]}$. Evidently, $\operatorname{supp}\left(k_{a}\right) \subset[-a, a]$. We introduce the function $\rho_{m}(x)=\mathrm{e}^{i x \Im\left(\lambda_{m}\right)} k_{a}(x)$ and we define

$$
\zeta_{m}=\frac{1}{\sqrt{2 \pi} \widehat{\rho}_{m}\left(i \bar{\lambda}_{m}\right)} \theta_{m} * \rho_{m} \quad\left(m \in \mathbb{Z}^{*}\right),
$$

where $\widehat{\rho}_{m}$ is the Fourier transform of $\rho_{m}$. Evidently, $\zeta_{m} \in L^{2}\left(-\frac{\widetilde{T}}{2}-a, \frac{\widetilde{T}}{2}+a\right)$. Let $T_{0}=\widetilde{T}+2 a$. From the convolution's properties, it follows that $\left(\zeta_{m}\right)_{m \in \mathbb{Z}^{*}}$ is a biorthogonal sequence to the family $\left(\mathrm{e}^{\lambda_{n} t}\right)_{n \in \mathbb{Z}^{*}}$ in $L^{2}\left(-\frac{T_{0}}{2}, \frac{T_{0}}{2}\right)$ and (3.31) is proved.

\section{Controllability Results}

Now we are able to prove the main result of this paper.

Proof of Theorem 1.1. Let $T>\max \left\{2 \beta, T_{0}\right\}$ and $\left(\zeta_{m}\right)_{m \in \mathbb{Z}^{*}}$ as in Theorem 3.11. We construct a control $v_{\varepsilon} \in$ $L^{2}(0, T)$ of $(1.13)$ corresponding to the initial data $\left(u^{0}, u^{1}\right) \in \mathcal{H}_{0}$ as follows

$$
v_{\varepsilon}(t)=-\sum_{m \in \mathbb{Z}^{*}} \frac{\mathrm{e}^{-\bar{\lambda}_{m} \frac{T}{2}}}{\widehat{f}_{|m|}}\left(\widehat{u}_{|m|}^{1}+\lambda_{m} \widehat{u}_{|m|}^{0}\right) \widetilde{\zeta}_{m}\left(t-\frac{T}{2}\right) \quad(t \in(0, T)),
$$


where $\widetilde{\zeta}_{m}$ is the extension by zero of $\zeta_{m}$ to the interval $\left(-\frac{T}{2}, \frac{T}{2}\right)$. From the properties of the biorthogonal sequence $\left(\zeta_{m}\right)_{m \in \mathbb{Z}^{*}}$, it is easy to see that $v_{\varepsilon}$ verifies (2.4). To conclude that $v_{\varepsilon}$ is a control for (1.13), we only have to prove that the right hand side of (4.1) converges in $L^{2}(0, T)$. This follows immediately from Theorem 3.11 and the fact that $\left(u^{0}, u^{1}\right) \in \mathcal{H}_{0}$. Indeed, we have that

$$
\begin{aligned}
& \int_{0}^{T}\left|v_{\varepsilon}(t)\right|^{2} \mathrm{~d} t=\int_{0}^{T}\left|-\sum_{m \in \mathbb{Z}^{*}} \frac{\mathrm{e}^{-\bar{\lambda}_{m} \frac{T}{2}}}{\widehat{f}_{|m|}}\left(\widehat{u}_{|m|}^{1}+\lambda_{m} \widehat{u}_{|m|}^{0}\right) \widetilde{\zeta}_{m}\left(t-\frac{T}{2}\right)\right|^{2} \mathrm{~d} t \\
= & \int_{-\frac{\tilde{T}}{2}-a}^{\frac{\tilde{T}}{2}+a}\left|-\sum_{m \in \mathbb{Z}^{*}} \frac{\mathrm{e}^{-\bar{\lambda}_{m} \frac{T}{2}}}{\widehat{f}_{|m|}}\left(\widehat{u}_{|m|}^{1}+\lambda_{m} \widehat{u}_{|m|}^{0}\right) \zeta_{m}(t)\right|^{2} \mathrm{~d} t \leq C\left(T_{0}\right)\left\|\left(u^{0}, u^{1}\right)\right\|_{\mathcal{H}_{0}}^{2} .
\end{aligned}
$$

The last inequality results from (3.31) with the constant $C\left(T_{0}\right)$ independent of $\varepsilon$ and $m$. Thus, the family of controls $\left(v_{\varepsilon}\right)_{\varepsilon \in(0,1)}$ is uniformly bounded in $L^{2}(0, T)$. In order to show that any weak limit of the family $\left(v_{\varepsilon}\right)_{\varepsilon \in(0,1)}$ is a control for (1.1) we only have to pass to the limit as $\varepsilon$ goes to zero in (2.4).

Remark 4.1. Theorem 1.1 ensures the existence of a time $T>0$, sufficiently large but independent of $\varepsilon$, for which the uniform controllability of (1.13) holds. From Propositions 3.2, 3.6 and 3.9 we can give an explicit expression of $T$. It is well known that the wave equation (1.1), corresponding to the limit case $\varepsilon=0$, is controllable in any time $T \geq 2 \pi$. Probably, the uniform controllability of (1.13) holds in the same time. However, to obtain the optimal time we should be able to obtain a multiplier function $M_{m}$ in Proposition 3.9 with arbitrarily small exponential type $L_{2}$ and constant $D$. This seems to be a difficult problem (see $[8,14,27]$ for similar uniform controllability results proved in a time larger than the optimal one).

\section{A. Appendix}

The aim of this section is to give the proof of Lemma 3.1 from Subsection 3.1. Through this section $C$ denotes an absolute positive constant.

Proof of Lemma 3.1. From the symmetry of the sequence $\left(\lambda_{n}\right)_{n \in \mathbb{Z}^{*}}$, it is sufficient to consider only the case $m \in \mathbb{N}^{*}$. We have that

$$
\begin{aligned}
& \prod_{\substack{n \in \mathbb{Z}^{*} \\
n \neq m}}\left|\frac{\lambda_{n}}{\lambda_{n}-\lambda_{m}}\right|^{2}=\frac{m^{2}+\varepsilon^{2} m^{4 \alpha}}{4 m^{2}} \prod_{\substack{n=1 \\
n \neq m}}^{\infty} \frac{\left(n^{2}+\varepsilon^{2} n^{4 \alpha}\right)^{2}}{\left[|n-m|^{2}+\varepsilon^{2}\left|n^{2 \alpha}-m^{2 \alpha}\right|^{2}\right]\left[(n+m)^{2}+\varepsilon^{2}\left|n^{2 \alpha}-m^{2 \alpha}\right|^{2}\right]} \\
= & \frac{m^{2}+\varepsilon^{2} m^{4 \alpha}}{4 m^{2}} \underbrace{\prod_{\substack{n=1 \\
n \neq m}}^{\infty} \frac{\left|\lambda_{n}\right|^{4}}{\left|\lambda_{|n-m|}\right|^{2}\left|\lambda_{m+n}\right|^{2}}}_{Q_{m}^{1}} \underbrace{\prod_{\substack{n=1 \\
n \neq m}}^{\infty} \frac{\left[|n-m|^{2}+\varepsilon^{2}|n-m|^{4 \alpha}\right]\left[(n+m)^{2}+\varepsilon^{2}(n+m)^{4 \alpha}\right]}{\left[|n-m|^{2}+\varepsilon^{2}\left|n^{2 \alpha}-m^{2 \alpha}\right|^{2}\right]\left[(n+m)^{2}+\varepsilon^{2}\left|n^{2 \alpha}-m^{2 \alpha}\right|^{2}\right]}}_{Q_{m}^{2}} .
\end{aligned}
$$

Since,

$$
Q_{m}^{1}=\prod_{\substack{n=1 \\ n \neq m}}^{\infty} \frac{\left|\lambda_{n}\right|^{4}}{\left|\lambda_{|n-m|}\right|^{2}\left|\lambda_{m+n}\right|^{2}} \leq \frac{\prod_{\substack{n=1 \\ n \neq m}}^{\infty}\left|\lambda_{n}\right|^{4}}{\prod_{n=1}^{m-1}\left|\lambda_{n}\right|^{2} \prod_{n=1}^{\infty}\left|\lambda_{n}\right|^{2} \prod_{\substack{n=m+1 \\ n \neq 2 m}}^{\infty}\left|\lambda_{n}\right|^{2}} \leq \frac{\left|\lambda_{2 m}\right|^{2}}{\left|\lambda_{m}\right|^{2}},
$$

it follows that

$$
Q_{m}^{1} \leq 16 \quad\left(m \in \mathbb{N}^{*}\right)
$$


For the product $Q_{m}^{2}$ we have that

$$
\begin{aligned}
Q_{m}^{2} & =\prod_{\substack{n=1 \\
n \neq m}}^{\infty}\left(1+\frac{|n-m|^{2}(n+m)^{2}\left[\varepsilon^{2} m^{4 \alpha-2} f\left(\frac{n}{m}\right)+\varepsilon^{4} m^{8 \alpha-4} g\left(\frac{n}{m}\right)\right]}{|n-m|^{2}(n+m)^{2}\left(1+\varepsilon^{2} \frac{\left|n^{2 \alpha}-m^{2 \alpha}\right|^{2}}{|n-m|^{2}}\right)\left(1+\varepsilon^{2} \frac{n^{2 \alpha}-\left.m^{2 \alpha}\right|^{2}}{(n+m)^{2}}\right)}\right) \\
& =\exp \left(\sum_{\substack{n=1 \\
n \neq m}}^{\infty} \ln \left(1+\frac{\varepsilon^{2} m^{4 \alpha-2} f\left(\frac{n}{m}\right)+\varepsilon^{4} m^{8 \alpha-4} g\left(\frac{n}{m}\right)}{\left(1+\varepsilon^{2} \frac{\left|n^{2 \alpha}-m^{2 \alpha}\right|^{2}}{|n-m|^{2}}\right)\left(1+\varepsilon^{2} \frac{n^{2 \alpha}-\left.m^{2 \alpha}\right|^{2}}{(n+m)^{2}}\right)}\right)\right) \\
& \leq \exp \left(\sum_{\substack{n=1 \\
n \neq m}}^{\infty} \frac{\varepsilon^{2} m^{4 \alpha-2} f\left(\frac{n}{m}\right)+\varepsilon^{4} m^{8 \alpha-4} g\left(\frac{n}{m}\right)}{\left(1+\varepsilon^{2} \frac{\left|n^{2 \alpha}-m^{2 \alpha}\right|^{2}}{|n-m|^{2}}\right)\left(1+\varepsilon^{2} \frac{\left|n^{2 \alpha}-m^{2 \alpha}\right|^{2}}{(n+m)^{2}}\right)}\right),
\end{aligned}
$$

where $f, g:(0, \infty) \rightarrow \mathbb{R}$ are the functions defined by

$$
\begin{gathered}
f(t)=|t-1|^{4 \alpha-2}+(t+1)^{4 \alpha-2}-2\left(t^{2}+1\right) \frac{\left(t^{2 \alpha}-1\right)^{2}}{\left(t^{2}-1\right)^{2}}, \\
g(t)=\left|t^{2}-1\right|^{4 \alpha-2}-\frac{\left(t^{2 \alpha}-1\right)^{4}}{\left(t^{2}-1\right)^{2}} .
\end{gathered}
$$

Let us remark that, for any $\alpha \in[0,1) \backslash\left\{\frac{1}{2}\right\}$, the function $g$ can be bounded as follows

$$
g(t) \leq C \begin{cases}t^{2 \alpha} & t<\frac{1}{2} \\ \left|t^{2}-1\right|^{4 \alpha-2} & t \in\left[\frac{1}{2}, 2\right] \\ t^{6 \alpha-4} & t>2\end{cases}
$$

We prove the following inequality

$$
S_{g}:=\sum_{\substack{n=1 \\ n \neq m}}^{\infty} \frac{\varepsilon^{4} m^{8 \alpha-4} g\left(\frac{n}{m}\right)}{\left(1+\varepsilon^{2} \frac{\left|n^{2 \alpha}-m^{2 \alpha}\right|^{2}}{|n-m|^{2}}\right)\left(1+\varepsilon^{2} \frac{\left|n^{2 \alpha}-m^{2 \alpha}\right|^{2}}{(n+m)^{2}}\right)} \leq C\left|\Re\left(\lambda_{m}\right)\right| \quad\left(m \in \mathbb{N}^{*}\right) .
$$

For that we write $S_{g}$ as follows

$$
S_{g}=\left(\sum_{n=1}^{\left[\frac{m}{2}\right]}+\sum_{\substack{n=\left[\frac{m}{2}\right]+1 \\ n \neq m}}^{2 m-1}+\sum_{n=2 m}^{\infty}\right) \frac{\varepsilon^{4} m^{8 \alpha-4} g\left(\frac{n}{m}\right)}{\left(1+\varepsilon^{2} \frac{\left|n^{2 \alpha}-m^{2 \alpha}\right|^{2}}{|n-m|^{2}}\right)\left(1+\varepsilon^{2} \frac{\left|n^{2 \alpha}-m^{2 \alpha}\right|^{2}}{(n+m)^{2}}\right)}=S_{g}^{1}+S_{g}^{2}+S_{g}^{3} .
$$

In order to evaluate $S_{g}^{i}$ we use the following inequalities

$$
\frac{\left|n^{2 \alpha}-m^{2 \alpha}\right|^{2}}{|n-m|^{2}} \geq|n-m|^{4 \alpha-2}, \quad \frac{\left|n^{2 \alpha}-m^{2 \alpha}\right|^{2}}{|n-m|^{2}} \geq(n+m)^{4 \alpha-2},
$$

which hold for every $\alpha \in\left(\frac{1}{2}, 1\right)$ and $n \in \mathbb{N}^{*}, n \neq m$. 
For any $\alpha \in[0,1) \backslash\left\{\frac{1}{2}\right\}$ we evaluate the sum $S_{g}^{1}$ by using (A.2) and by taking into account that $m+n \leq 2 m$ and $m-n \geq \frac{1}{2} m$ for every $n \in\left[1, \frac{m}{2}\right]$. We deduce that

$$
S_{g}^{1} \leq C \sum_{n=1}^{\left[\frac{m}{2}\right]} \frac{\varepsilon^{4} m^{6 \alpha-4} n^{2 \alpha}}{\left(1+\varepsilon^{2} m^{4 \alpha-2}\right)^{2}} \leq \frac{C \varepsilon^{4} m^{6 \alpha-4}}{\left(1+\varepsilon^{2} m^{4 \alpha-2}\right)^{2}} \int_{1}^{\left[\frac{m}{2}\right]+1} t^{2 \alpha} \mathrm{d} t \leq \frac{C \varepsilon^{4} m^{8 \alpha-3}}{\left(1+\varepsilon^{2} m^{4 \alpha-2}\right)^{2}} \leq C\left|\Re\left(\lambda_{m}\right)\right| .
$$

Similarly, for any $\alpha \in[0,1) \backslash\left\{\frac{1}{2}\right\}$ we evaluate the sum $S_{g}^{3}$ as follows

$$
S_{g}^{3} \leq \sum_{n=2 m}^{\infty} \frac{C \varepsilon^{4} m^{2 \alpha} n^{6 \alpha-4}}{\left(1+\varepsilon^{2} \frac{\left|n^{2 \alpha}-m^{2 \alpha}\right|^{2}}{|n-m|^{2}}\right)\left(1+\varepsilon^{2} \frac{\left|n^{2 \alpha}-m^{2 \alpha}\right|^{2}}{(n+m)^{2}}\right)} \leq \sum_{n=2 m}^{\infty} \frac{C \varepsilon^{4} m^{2 \alpha} n^{6 \alpha-4}}{\left(1+\varepsilon^{2} n^{4 \alpha-2}\right)^{2}} \leq C\left|\Re\left(\lambda_{m}\right)\right| .
$$

For $\alpha \in\left[0, \frac{1}{2}\right)$ we analyze $S_{g}^{2}$ as follows

$$
S_{g}^{2} \leq \sum_{n=\left[\frac{m}{2}\right]+1}^{2 m-1} \frac{C \varepsilon^{4}\left|n^{2}-m^{2}\right|^{4 \alpha-2}}{\left(1+\varepsilon^{2} \frac{\left|n^{2 \alpha}-m^{2 \alpha}\right|^{2}}{|n-m|^{2}}\right)\left(1+\varepsilon^{2} \frac{\left|n^{2 \alpha}-m^{2 \alpha}\right|^{2}}{(n+m)^{2}}\right)} \leq \sum_{n=\left[\frac{m}{2}\right]+1}^{2 m-1} C \varepsilon^{4} m^{4 \alpha-2}|n-m|^{4 \alpha-2} \leq C\left|\Re\left(\lambda_{m}\right)\right| .
$$

And, for $\alpha \in\left(\frac{1}{2}, 1\right)$, we have to treat separately the cases $m \leq \gamma_{\varepsilon}$ and $m>\gamma_{\varepsilon}$. For $m \leq \gamma_{\varepsilon}$, we notice that the function $g$ is continuous on a compact set, so there exists a positive constant $C$ independent of $\varepsilon$ and $m$ such that $g(t) \leq C$. By using again (A.4) it follows that

$$
S_{g}^{2} \leq \sum_{n=\left[\frac{m}{2}\right]+1}^{2 m-1} \frac{C \varepsilon^{4} m^{8 \alpha-4}}{\left(1+\varepsilon^{2}|n-m|^{4 \alpha-2}\right)\left(1+\varepsilon^{2} \frac{|n-m|^{4 \alpha}}{m^{2}}\right)} \leq \sum_{k=1}^{m} \frac{C \varepsilon^{4} m^{8 \alpha-2}}{\left(1+\varepsilon^{2} k^{4 \alpha-2}\right)\left(m^{2}+\varepsilon^{2} k^{4 \alpha}\right)} \leq C\left|\Re\left(\lambda_{m}\right)\right| .
$$

For the case $m>\gamma_{\varepsilon}$ it follows that

$$
\begin{aligned}
S_{g}^{2} & \leq \sum_{n=\left[\frac{m}{2}\right]+1}^{2 m-1} \frac{C \varepsilon^{4} m^{4 \alpha-2}|n-m|^{4 \alpha-2}}{\varepsilon^{2} m^{4 \alpha-2}\left(1+\varepsilon^{2} \frac{(m+n)^{4 \alpha-2}|n-m|^{2}}{m^{2}}\right)} \leq \sum_{k=1}^{m} \frac{C \varepsilon^{2} m^{2} k^{4 \alpha-2}}{m^{2}+\varepsilon^{2} m^{4 \alpha-2} k^{2}} \\
& \leq C \varepsilon^{2} \sum_{k=1}^{\left[\frac{m^{2-2 \alpha}}{\varepsilon}\right]} k^{4 \alpha-2}+C m^{4-4 \alpha} \sum_{k=\left[\frac{m^{2-2 \alpha}}{\varepsilon}\right]+1}^{m} k^{4 \alpha-4} \leq C\left|\Re\left(\lambda_{m}\right)\right|,
\end{aligned}
$$

which concludes the proof of (A.3).

Let us remark that, for any $\alpha \in[0,1) \backslash\left\{\frac{1}{2}\right\}$, the function $f$ can be bounded in the following way

$$
f(t) \leq C \begin{cases}t^{2 \alpha} & t<\frac{1}{2} \\ |t-1|^{4 \alpha-2} & t \in\left[\frac{1}{2}, 2\right] \\ t^{2 \alpha-2} & t>2\end{cases}
$$

We prove the following inequality

$$
S_{f}:=\sum_{\substack{n=1 \\ n \neq m}}^{\infty} \frac{\varepsilon^{2} m^{4 \alpha-2} f\left(\frac{n}{m}\right)}{\left(1+\varepsilon^{2} \frac{\left|n^{2 \alpha}-m^{2 \alpha}\right|^{2}}{|n-m|^{2}}\right)\left(1+\varepsilon^{2} \frac{\left|n^{2 \alpha}-m^{2 \alpha}\right|^{2}}{(n+m)^{2}}\right)} \leq C\left|\Re\left(\lambda_{m}\right)\right| \quad\left(m \in \mathbb{N}^{*}\right) .
$$


Indeed, we have that

$$
S_{f}=\left(\sum_{n=1}^{\left[\frac{m}{2}\right]}+\sum_{\substack{n=\left[\frac{m}{2}\right]+1 \\ n \neq m}}^{2 m-1}+\sum_{n=2 m}^{\infty}\right) \frac{C \varepsilon^{2} m^{4 \alpha-2} f\left(\frac{n}{m}\right)}{\left(1+\varepsilon^{2} \frac{\left|n^{2 \alpha}-m^{2 \alpha}\right|^{2}}{|n-m|^{2}}\right)\left(1+\varepsilon^{2} \frac{\left|n^{2 \alpha}-m^{2 \alpha}\right|^{2}}{(n+m)^{2}}\right)}=S_{f}^{1}+S_{f}^{2}+S_{f}^{3} .
$$

For any $\alpha \in[0,1) \backslash\left\{\frac{1}{2}\right\}$ we evaluate the sum $S_{f}^{1}$ by taking into account (A.5) and the fact that $m+n \leq 2 m$ and $m-n \geq \frac{1}{2} m$ for every $n \in\left[1, \frac{m}{2}\right]$. We deduce that

$$
S_{f}^{1}=\sum_{n=1}^{\left[\frac{m}{2}\right]} \frac{C \varepsilon^{2} m^{2 \alpha-2} n^{2 \alpha}}{\left(1+\varepsilon^{2} \frac{\left(m^{2 \alpha}-n^{2 \alpha}\right)^{2}}{(m-n)^{2}}\right)\left(1+\varepsilon^{2} \frac{\left(m^{2 \alpha}-n^{2 \alpha}\right)^{2}}{(n+m)^{2}}\right)} \leq \frac{C \varepsilon^{2} m^{2 \alpha-2}}{\left(1+\varepsilon^{2} m^{4 \alpha-2}\right)^{2}} \sum_{n=1}^{\left[\frac{m}{2}\right]} n^{2 \alpha} \leq C\left|\Re\left(\lambda_{m}\right)\right| .
$$

Similarly, for any $\alpha \in[0,1) \backslash\left\{\frac{1}{2}\right\}$, we deduce that $S_{f}^{3}$ is bounded by $C\left|\Re\left(\lambda_{m}\right)\right|$. Indeed,

$$
S_{f}^{3}=\sum_{n=2 m}^{\infty} \frac{\varepsilon^{2} m^{2 \alpha} n^{2 \alpha-2}}{\left(1+\varepsilon^{2} \frac{\left|n^{2 \alpha}-m^{2 \alpha}\right|^{2}}{|n-m|^{2}}\right)\left(1+\varepsilon^{2} \frac{\left|n^{2 \alpha}-m^{2 \alpha}\right|^{2}}{(n+m)^{2}}\right)} \leq \sum_{n=2 m}^{\infty} \frac{\varepsilon^{2} m^{2 \alpha} n^{2 \alpha-2}}{\left(1+\varepsilon^{2} n^{4 \alpha-2}\right)^{2}} \leq C\left|\Re\left(\lambda_{m}\right)\right| .
$$

For $\alpha \in\left[0, \frac{1}{2}\right)$ we evaluate $S_{f}^{2}$, as follows

$$
\begin{aligned}
S_{f}^{2} & =\sum_{n=\left[\frac{m}{2}\right]+1}^{2 m-1} \frac{C \varepsilon^{2}|n-m|^{4 \alpha-2}}{\left(1+\varepsilon^{2} \frac{\left|n^{2 \alpha}-m^{2 \alpha}\right|^{2}}{|n-m|^{2}}\right)\left(1+\varepsilon^{2} \frac{\left|n^{2 \alpha}-m^{2 \alpha}\right|^{2}}{(n+m)^{2}}\right)} \leq \sum_{n=\left[\frac{m}{2}\right]+1}^{2 m-1} \frac{C \varepsilon^{2}|n-m|^{4 \alpha-2}}{1+\varepsilon^{2} \frac{\left|n^{2 \alpha}-m^{2 \alpha}\right|^{2}}{|n-m|^{2}}} \\
& \leq \sum_{n=\left[\frac{m}{2}\right]+1}^{2 m-1} \frac{C \varepsilon^{2}|n-m|^{4 \alpha}}{|n-m|^{2}+\varepsilon^{2} m^{4 \alpha}}=\sum_{k=1}^{m} \frac{C \varepsilon^{2} k^{4 \alpha}}{k^{2}+\varepsilon^{2} m^{4 \alpha}} \leq \sum_{k=1}^{m} \frac{C \varepsilon^{2} m^{4 \alpha}}{k^{2}+\varepsilon^{2} m^{4 \alpha}} \leq C\left|\Re\left(\lambda_{m}\right)\right| .
\end{aligned}
$$

If $\alpha \in\left(\frac{1}{2}, 1\right)$ and $m \leq \gamma_{\varepsilon}$, we have that

$$
S_{f}^{2} \leq \sum_{n=\left[\frac{m}{2}\right]+1}^{2 m-1} \frac{C \varepsilon^{2}|n-m|^{4 \alpha-2} m^{2}}{\left(1+\varepsilon^{2} m^{4 \alpha-2}\right)\left(m^{2}+\varepsilon^{2}|n-m|^{2} m^{4 \alpha-2}\right)} \leq C \varepsilon^{2} m^{2} \sum_{k=1}^{m} k^{4 \alpha-2} \leq C\left|\Re\left(\lambda_{m}\right)\right|,
$$

and for $\alpha \in\left(\frac{1}{2}, 1\right)$ and $m>\gamma_{\varepsilon}$ the following estimates takes place

$$
\begin{aligned}
S_{f}^{2} & \leq \sum_{n=\left[\frac{m}{2}\right]+1}^{2 m-1} \frac{C \varepsilon^{2}|n-m|^{4 \alpha-2} m^{2}}{\left(1+\varepsilon^{2} m^{4 \alpha-2}\right)\left(m^{2}+\varepsilon^{2}|n-m|^{2} m^{4 \alpha-2}\right)} \leq \frac{C \varepsilon^{2} m^{2}}{1+\varepsilon^{2} m^{4 \alpha-2}} \sum_{k=1}^{m} \frac{k^{4 \alpha-2}}{m^{2}+\varepsilon^{2} k^{2} m^{4 \alpha-2}} \\
& \leq C m^{4-4 \alpha}\left(\sum_{k=1}^{\left[\frac{m^{2-2 \alpha}}{\varepsilon}\right]} \frac{k^{4 \alpha-2}}{m^{2}}+\sum_{k=\left[\frac{m^{2}-2 \alpha}{\varepsilon}\right]+1}^{m} \frac{k^{4 \alpha-4}}{\varepsilon^{2} m^{4 \alpha-2}}\right) \leq C\left|\Re\left(\lambda_{m}\right)\right| .
\end{aligned}
$$

Now from (A.1), (A.3) and (A.6) it results (3.2) and the proof of Lemma 3.1 ends.

Acknowledgements. The first author was partially supported by the strategic grant POSDRU/CPP107/ DMI1.5/S/78421, Project ID 78421 (2010), co-financed by the European Social Fund - Investing in People, within the Sectorial Operational Programme Human Resources Development 2007-2013. The second author was partially supported by Grant PN-II-ID-PCE-2011-3-0257 of the Romanian National Authority for Scientific Research, CNCS UEFISCDI and by Grant MTM2011-29306 funded by MICINN (Spain). 


\section{REFERENCES}

[1] S.A. Avdonin and S.A. Ivanov, Families of exponentials. The method of moments in controllability problems for distributed parameter systems. Cambridge University Press (1995).

[2] C. Brändle, E. Colorado, A. de Pablo and U. Sánchez, A concave-convex elliptic problem involving the fractional Laplacian. Proc. Roy. Soc. Edinburgh Sect. A 143 (2013) 39-71.

[3] L. Caffarelli and L. Silvestre, An extension problem related to the fractional Laplacian. Commun. Partial Differ. Eqs. 32 (2007) 1245-1260.

[4] T. Cazenave and A. Haraux, An Introduction to Semilinear Evolution Equation. Oxford University Press Inc., New York (1998).

[5] S. Chen and R. Triggiani, Proof of Extensions of Two Conjectures on Structural Damping for Elastic Systems. Pacific J. Math. 136 (1989) $15-55$.

[6] S. Chen and R. Triggiani, Characterization of Domains of Fractional Powers of Certain Operators Arising in Elastic Systems and Applications. J. Differ. Eqs. 88 (1990) 279-293.

[7] J.M. Coron, Control and nonlinearity, Mathematical Surveys and Monographs. Amer. Math. Soc. Providence, RI 136 (2007).

[8] J.M. Coron and S. Guerrero, Singular optimal control: a linear 1-D parabolic-hyperbolic example. Asymptot. Anal. 44 (2005) $237-257$.

[9] Q.-Y. Guan and Z.-M. Ma, Boundary problems for fractional Laplacians. Stoch. Dyn. 5 (2005) 385-424.

[10] R.J. DiPerna, Convergence of approximate solutions to conservation laws. Arch. Ration. Mech. Anal. 82 (1983) $27-70$.

[11] J. Edward, Ingham-type inequalities for complex frequencies and applications to control theory. J. Math. Appl. 324 (2006) 941-954.

[12] H.O. Fattorini and D.L. Russell, Uniform bounds on biorthogonal functions for real exponentials with an application to the control theory of parabolic equations. Q. Appl. Math. 32 (1974/75) 45-69.

[13] H.O. Fattorini and D.L. Russell, Exact controllability theorems for linear parabolic equations in one space dimension. Arch. Ration. Mech. Anal. 43 (1971) 272-292.

[14] O. Glass, A complex-analytic approach to the problem of uniform controllability of a transport equation in the vanishing viscosity limit. J. Funct. Anal. 258 (2010) 852-868.

[15] S.W. Hansen, Bounds on Functions Biorthogonal to Sets of Complex Exponentials; Control of Dumped Elastic Systems. J. Math. Anal. Appl. 158 (1991) 487-508.

[16] L. Ignat and E. Zuazua, Dispersive Properties of Numerical Schemes for Nonlinear Schrödinger Equation, Foundations of Computational Mathematics, Santander 2005, London Math.l Soc. Lect. Notes. Edited by L.M. Pardo. Cambridge University Press 331 (2006) 181-207.

[17] L. Ignat and E. Zuazua, Numerical dispersive schemes for the nonlinear Schrödinger equation. SIAM J. Numer. Anal. 47 (2009) 1366-1390.

[18] C. Imbert, A non-local regularization of first order Hamilton-Jacobi equations, J. Differ. Eqs. 211 (2005) $218-246$.

[19] A.E. Ingham, A note on Fourier transform. J. London Math. Soc. 9 (1934) 29-32.

[20] A.E. Ingham, Some trigonometric inequalities with applications to the theory of series Math. Zeits. 41 (1936) $367-379$.

[21] A. Khapalov, Approximate controllability and its well-posedness for the semilinear reaction-diffusion equation with internal lumped controls. ESAIM: COCV 4 (1999) 83-98.

[22] V. Komornik and P. Loreti, Fourier Series in Control Theory. Springer-Verlag, New-York (2005).

[23] M. Léautaud, Uniform controllability of scalar conservation laws in the vanishing viscosity limit. SIAM J. Control Optim. 50 (2012) 1661-1699.

[24] A. López, X. Zhang and E. Zuazua, Null controllability of the heat equation as singular limit of the exact controllability of dissipative wave equation. J. Math. Pures Appl. 79 (2000) 741-808.

[25] S. Micu, J.H. Ortega and A.F. Pazoto, Null-controllability of a Hyperbolic Equation as Singular Limit of Parabolic Ones. J. Fourier Anal. Appl. 41 (2010) 991-1007.

[26] S. Micu and I. Rovența, Uniform controllability of the linear one dimensional Schrödinger equation with vanishing viscosity. ESAIM: COCV 18 (2012) 277-293.

[27] S. Micu and L. de Teresa, A spectral study of the boundary controllability of the linear 2-D wave equation in a rectangle, Asymptot. Anal. 66 (2010) 139-160.

[28] L. Miller, Controllability cost of conservative systems: resolvent condition and transmutation. J. Funct. Anal. 218 (2005) 425-444.

[29] R.E.A.C. Paley and N. Wiener, Fourier Transforms in Complex Domains. AMS Colloq. Publ. Amer. Math. Soc. New-York 19 (1934).

[30] L. Rosier and P. Rouchon, On the Controllability of a Wave Equation with Structural Damping. Int. J. Tomogr. Stat. 5 (2007) 79-84.

[31] D.L. Russel, A unified boundary controllability theory for hyperbolic and parabolic partial differential equation. Stud. Appl. Math. 52 (1973) 189-221.

[32] T.I. Seidman, On uniform nullcontrollability and blow-up estimates, Chapter 15 in Control Theory of Partial Differential Equations, edited by O. Imanuvilov, G. Leugering, R. Triggiani and B.Y. Zhang. Chapman and Hall/CRC, Boca Raton (2005) $215-227$. 
[33] O. Szász, Über die Approximation stetiger Funktionen durch lineare Aggregate von Potenzen. Math. Ann. 77 (1916) $482-496$.

[34] M. Tucsnak and G. Weiss, Observation and Control for Operator Semigroups. Birkhuser Advanced Texts. Springer, Basel (2009).

[35] R.M. Young, An Introduction to Nonharmonic Fourier Series. Academic Press, New-York (1980).

[36] J. Zabczyk, Mathematical Control Theory: An Introduction. Birkhuser, Basel (1992).

[37] E. Zuazua, Propagation, Observation, Control and Numerical Approximation of Waves approximated by finite difference methods. SIAM Rev. 47 (2005) 197-243. 